\title{
Nonlinear electrostatic effects in MEMS ring-based rate sensors under shock excitation
}

\author{
Stefan Sieberer ${ }^{\mathrm{a}, *}$, Stewart McWilliam ${ }^{\mathrm{b}}$, Atanas A. Popov ${ }^{\mathrm{b}}$ \\ ${ }^{a}$ Johannes Kepler University Linz, Altenberger Strasse 69, 4040 Linz, Austria \\ ${ }^{b}$ University of Nottingham, University Park, NG7 2RD, Nottingham, United Kingdom
}

\begin{abstract}
The vibration response of a capacitive ring-based Coriolis Vibrating Gyroscope (CVG) subjected to in-plane shock is modelled and analysed to quantify the effect of shock on angular velocity measurement. The model developed considers a ring resonator with 8 uniformly spaced support legs and describes the in-plane ring response as the sum of the first 3 modes of a perfect ring and the nonlinear electrostatic force as a Taylor series. When a severe in-plane shock is applied, the rigid body response of the ring reduces the electrode gap significantly and a high order expansion is needed to represent the electrostatic force. These nonlinear forces are shown to cause direct and mixed mode coupling to occur, which can significantly modify the response characteristics. Numerical results are presented and interpreted for a range of shock cases to demonstrate the importance of mode coupling, and estimates are made to quantify the angular rate measurement error caused by shock for devices based on $2 \theta$ - and $3 \theta$-modes of operation. To aid the design of devices that are more resilient to shock, a parameter study is performed to identify the modal frequency ratios that minimise this coupling.
\end{abstract}

Keywords: Micro Electro Mechanical System (MEMS), Ring resonator, Gyroscopic sensor, Shock sensitivity analysis, Nonlinear electrostatics, Modal coupling

\section{Background and introduction}

Micro-engineered CVGs are present in an increasingly wide range of applications, where their small size and low cost provide a significant advantage compared to macroscale devices. For applications such as in-flight inertial guidance and control of aircraft and space satellites, the sensors operate in harsh environmental conditions [26] and high accuracy measurements are required, particularly in the presence of external shock inputs. Micro ring-based CVGs use flexural ring vibration to detect angular rate and are well suited to shock environments compared to other gyro types because the flexural ring modes are unaffected by external shock inputs under linear conditions [9]. This paper investigates the influence of nonlinear electrostatic forces on the in-plane response of these sensors under severe shock conditions. A mathematical model of the ring resonator is developed to highlight the different types of nonlinear coupling and simulate the resulting response under shock conditions.

\footnotetext{
${ }^{*}$ Corresponding author

Email address: stefan.sieberer@jku.at (Stefan Sieberer)
} 
Figure 1 shows the dominant in-plane modes of a ring that contribute to the dynamics of a vibrating ring-based rate sensor. The modes of vibration for a perfect ring occur in degenerate pairs [8] having the same frequency and indeterminate mode orientation. Figure 1(a) shows one of the rigid-body (10-) modes, whilst Figures 1(b) and $1(\mathrm{c})$ show one of the two flexural $2 \theta$ - and $3 \theta$-modes, respectively. In ring-based CVGs the in-plane $2 \theta$ - or $3 \theta$-flexural vibrations are used to detect angular rate applied about the polar ring axis, and this is achieved by driving one of the modes into resonance and measuring the response amplitude of the companion mode, which is proportional to the angular rate. For state-of-the-art devices, drive forces and response measurements are achieved electrostatically using curved electrodes located around the ring (see Figure 2). Under severe shock conditions, rigid-body motion of the ring on its supports causes the gap between the ring and electrodes to vary significantly around the ring circumference and induce nonlinear electrostatic forces to act on the ring. The applied shock induces nonlinear coupling between the rigid-body and flexural modes of the ring, and a consequence of this coupling is that severe shock responses can induce flexural responses in the ring. Given that the vibration is used to measure angular rate, the shock-induced flexural response may be misinterpreted by the sensor as angular rate causing measurement inaccuracies. The motivation for this paper is to model the nonlinear coupling arising from the electrostatics in detail to quantify and better understand its influence on sensor performance.

Previous work on the shock response of micro-engineered or MEMS (Micro-ElectroMechanical-Systems) often describe the resulting response as being quasi-static [27], where the shock pulse is much longer than half the time period of natural oscillation. More recently, the dynamic response of micro-structures in shock conditions has been reported by Younis and co-workers $[37,36,35,19]$ who studied electrostatically actuated micro-beams under large amplitude shock excitation. The severe shock response of electrostatically operated ring based resonators, like those used in vibrating ring rate sensors, has had little attention in the research literature. These resonators are normally designed to have low levels of damping to reduce energy consumption, but most research on shock considers responses under critical damping conditions [16, 29], like those typically experienced in micro-accelerometers incorporating micro-beams.
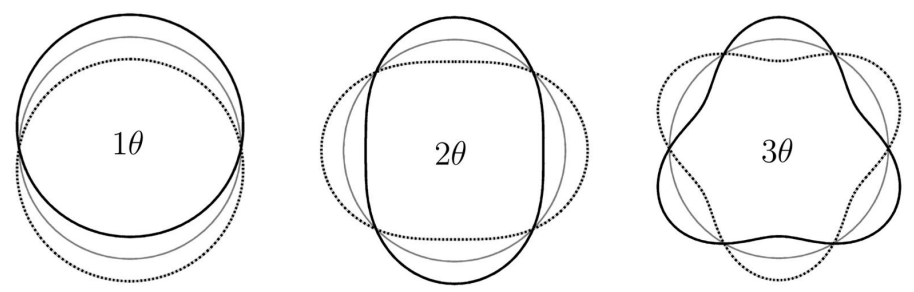

Figure 1: In-plane rigid-body and flexural ring modes.

The behaviour of micro-ring resonators under standard 'no shock' operating conditions is well understood and much attention has focused on enhancing performance by trimming the in-plane $[15,10,4]$ and out-of-plane [7,21] frequencies. Serandour et al. [23] studied nonlinear geometric and electrostatic effects in vibrating ring-based resonators, but did not consider shock response. Yoon et al. [34] analysed the influence of electrostatic nonlinearities on motion detection for capacitive ring-resonators under external 
vibration, but linearised the electrostatic forces and only considered the sensing process as being nonlinear. Sieberer et al. [25] analysed the in-plane shock response of an electrostatically operated ring by representing the ring response in terms of the $1 \theta$ - and $2 \theta$-modes of vibration for a perfect ring, and included quadratic nonlinear forces in the equations of motion. However, the analysis was restricted to quadratic electrostatic terms. The current paper extends this approach to include higher order electrostatic terms when the ring response is expressed as the sum of the rigid body $\left(1 \theta_{-}\right)$and flexural $\left(2 \theta-\right.$ and $\left.3 \theta_{-}\right)$ modes of a perfect ring with ideal supports. Different levels of approximation (linear, quadratic, cubic, etc.) are used to model the nonlinear electrostatic forces and the developed model is used to calculate the in-plane ring response via numerical simulation. The model developed is similar to Chouvion [5], which showed that severe shocks can cause the vibrating response to jump to another stable state under certain circumstances, but focuses on the 8 support leg case and presents the resulting equations of motion in detail together with numerical results to explicitly demonstrate the importance of direct and mixed mode coupling. Calculations are also performed to quantify angular rate measurement error caused by the nonlinear coupling mechanism, for devices operating using $2 \theta$ - and $3 \theta$-vibrations. This is achieved by converting the induced flexural $2 \theta$-response into an equivalent rate output and comparing the results against typical noise levels for a sensor.

The shock-induced response of a typical ring design is investigated by numerically simulating the governing equations of motion. Different electrode configurations are considered to highlight the difference in shock performance achieved using both inner and outer electrodes compared to using outer electrodes only [3,2,33]. Also to aid the design of devices that are resilient to shock, parameter studies are performed to investigates how the ratio of $2 \theta$ - to $3 \theta$-frequencies affects the resulting shock induced response, for devices operating using $2 \theta$ - and $3 \theta$-vibrations.

The paper is organised as follows. The equations of motion governing the in-plane shock response of a vibrating ring rate sensor with 8 support legs under electrostatic loading are developed in Section 2. Numerical results for the shock response are presented in Section 3, including the impact of using different electrostatic configurations in Sections 3.2 and 3.4, together with a brief convergence study in Section 3.5. Section 3.6 converts the flexural response produced by nonlinear mode coupling from shock to an equivalent angular rate of the sensor, and Section 3.7 presents a parameter study showing the influence of different resonator frequencies on the induced shock response.

\section{Ring resonator modelling}

A linear mechanical model of a ring resonator with 8 uniformly spaced flexible supports that is surrounded by capacitive electrodes is developed in this section. The in-plane displacements of the ring are described in terms of the mode shapes for a perfect ring (see Figure 1). For axisymmetric structures, like rings, modes occur in pairs having the same natural frequency and are aligned orthogonally to form a degenerate pair of modes [30].

Figure 2 shows a schematic diagram for a capacitively operated CVG. The ring resonator (1) is supported by flexible legs (2) and surrounded by electrodes, used for capacitive actuation and sensing (3). The support legs are attached to a rigid base (4) 


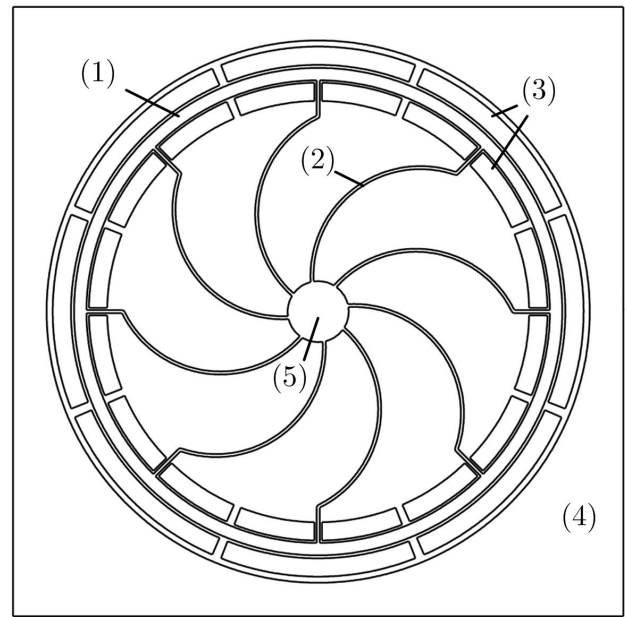

Figure 2: Ring layer displaying: the ring (1), support legs (2), a central hub (5), and electrodes (3). The base is indicated by (4).

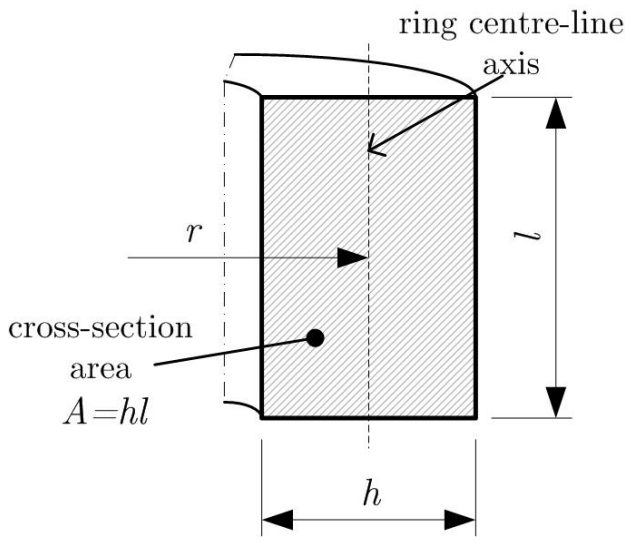

Figure 3: A section of the ring with the centre-line axis, the mean radius $r$, the axial height $l$, the radial width $h$, and the ring cross-sectional area $A$.

via central hub (5). Under standard operating conditions, capacitive forces are used to excite the ring resonator and under shock conditions elastic deformations in the supports change the radial spacing between the ring and surrounding electrodes, modifying the capacitor gap and resultant electrostatic forcing.

The ring resonator is modelled as a uniform, thin, perfect ring, and Figure 3 shows a section of the ring with mean radius $r$, radial thickness $h$, axial length $l$, and crosssectional area $A=h l$. The in-plane ring motion is limited by the capacitor gap size, which is much smaller than the ring radial thickness. Given that the rigid-body displacement and elastic deformation of the ring is small, a linear model of the ring and supports is used to describe the ring motion [1]. For the purposes of analysis, the ring motion is expressed in terms of the responses of the modes of an unsupported perfect ring, as shown in Figure 1. Table 1 summarises the notation used to describe the generalised coordinates associated with the different ring mode-shape functions.

The support legs connecting the ring and hub consist of thin beam structures, which can be modelled as radial and tangential linear springs for the assumed range of ring deflections. The shock excitation is included in the model as base excitation applied to the central hub, and the equations of motion of the system are obtained using Lagrange's equation.

Assuming inextensional ring motion, the radial and tangential displacements, $w$ and $v$ respectively (see Figure 4 ) of the ring relative to the hub at ring angle $\varphi$ satisfy and are given by [31]:

$$
\begin{gathered}
w(\varphi)=P_{1} \cos (\varphi)+P_{2} \sin (\varphi)+Q_{1} \cos (2 \varphi)+Q_{2} \sin (2 \varphi)+R_{1} \cos (3 \varphi)+R_{2} \sin (3 \varphi) \\
v(\varphi)=P_{1} \sin (\varphi)-P_{2} \sin (\varphi)+\frac{Q_{1}}{2} \sin (2 \varphi)-\frac{Q_{2}}{2} \sin (2 \varphi)+\frac{R_{1}}{3} \sin (3 \varphi)-\frac{R_{2}}{3} \sin (3 \varphi)
\end{gathered}
$$




\begin{tabular}{ccc}
$\begin{array}{c}\text { Mode of } \\
\text { vibration }\end{array}$ & $\begin{array}{c}\text { Amplitudes of gen- } \\
\text { eralised coordinates }\end{array}$ & $\begin{array}{c}\text { Mode } \\
\text { shape function }\end{array}$ \\
\hline $1 \theta$ & $P_{1}$ & $\cos (\varphi)$ \\
$1 \theta$ & $P_{2}$ & $\sin (\varphi)$ \\
$2 \theta$ & $Q_{1}$ & $\cos (2 \varphi)$ \\
$2 \theta$ & $Q_{2}$ & $\sin (2 \varphi)$ \\
$3 \theta$ & $R_{1}$ & $\cos (3 \varphi)$ \\
$3 \theta$ & $R_{2}$ & $\sin (3 \varphi)$ \\
\hline
\end{tabular}

Table 1: Generalised coordinates for the $1 \theta-, 2 \theta-$, and $3 \theta$-modes of vibration, and mode shape function dependent on the ring angle $\varphi$ (see Figure 4).

These displacements are the sums of the modal radial and tangential displacements and are dependent on the angular position on the ring, described by the ring angle $\varphi$ which is shown in Figure 4. Note that the factors $1 / 2$ and $1 / 3$ accompanying $2 \theta$ - and $3 \theta$ flexural generalised coordinates of the tangential displacement, respectively, arise from the assumption of linear inextensional flexural ring vibration.

The radial and tangential displacements of the ring relative to the hub are defined by Equations (1a) and (1b). These expressions are combined with the hub displacements $x_{b}$ and $y_{b}$ shown in Figure 4 and then used to obtain energy expressions for the resonator, required in Lagrange's equations. The relevant energy expressions are provided in the next section.

\subsection{Energies of the mechanical resonator}

In this paper Lagrange's equation is used to derive the equations of motion governing the rigid-body and flexural in-plane deformation of the ring based on the generalised coordinates summarised in Table 1. Lagrange's equation is based on the energies of the system and is given by

$$
\frac{\mathrm{d}}{\mathrm{d} t}\left(\frac{\partial T}{\partial \dot{q}_{j}}\right)-\frac{\partial T}{\partial q_{j}}+\frac{\partial D}{\partial \dot{q}_{j}}+\frac{\partial(U+W)}{\partial q_{j}}=\frac{\partial E_{\mathrm{cap}}}{\partial q_{j}}, \quad(j=1,2, \cdots, n)
$$

where $T$ is the kinetic energy of the ring, $U$ is the strain energy of the ring, $W$ is the potential energy of deformation of the support structure, $D$ is the Rayleigh dissipation function and $E_{\text {cap }}$ is the electrostatic potential energy. Each of the mechanical quantities is considered in this section. The electrostatic potential energy is considered in detail in Section 2.3.

\subsubsection{Kinetic energy of the ring}

Figure 4 shows the components of absolute motion of the hub $\left(x_{b}\right.$ and $\left.y_{b}\right)$ and the relative motions of the ring relative to the hub $(w, v)$. These components can be combined easily to obtain the absolute displacement $(x, y)$ of the element of the ring at ring angle $\varphi$. Taking the time derivative, it can be shown easily that the components of absolute velocity $(\dot{x}$ and $\dot{y})$ of the ring element are given by:

$$
\begin{aligned}
\dot{x} & =\dot{w} \cos (\varphi)+\dot{v} \sin (\varphi)+\dot{x}_{b} \\
\dot{y} & =\dot{v} \cos (\varphi)-\dot{w} \sin (\varphi)+\dot{y}_{b}
\end{aligned}
$$


where a dot implies differentiation with respect to time.

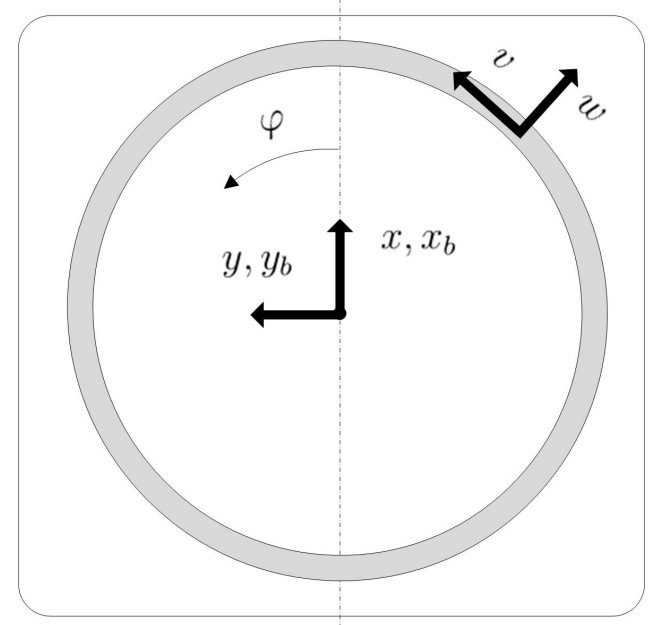

Figure 4: The absolute Cartesian coordinate system $x-y$, the base motion $x_{b}-y_{b}$, the local, relative ring motion $v$-w, and the ring angle $\varphi$. The angle $\varphi=0$ is aligned with the $x$-axis.

The kinetic energy of the ring is given by:

$$
T=\frac{1}{2} \int_{0}^{2 \pi}\left(\dot{x}^{2}+\dot{y}^{2}\right) A \rho r \mathrm{~d} \varphi
$$

where $\rho$ is the density of the ring material.

Using Equations (3a) and (3b) in Equation (4) and integrating it can be shown that the kinetic energy of the ring is given by:

$$
T=\frac{m}{2}\left[\left(\dot{P}_{1}+\dot{x}_{b}\right)^{2}+\left(\dot{P}_{2}-\dot{y}_{b}\right)^{2}+\frac{5}{8}\left({\dot{Q_{1}}}^{2}+{\dot{Q_{2}}}^{2}\right)+\frac{10}{18}\left({\dot{R_{1}}}^{2}+{\dot{R_{2}}}^{2}\right)\right]
$$

where $m$ is the physical ring mass. The kinetic energy expression includes contributions from the ring rigid-body motion and flexural in-plane vibrations relative to the ring, as well as the base excitation. Equation (5) indicates that the generalised coordinates of the ring are not coupled in the equation for kinetic energy.

\subsubsection{Strain energy of the ring}

As the in-plane displacement of the ring is small compared to the radial thickness of the structure, the ring can be considered to be inextensible and linear, and the bending strain energy $U$ can be expressed as [31]:

$$
U=\frac{E I_{c}}{2 r^{3}} \int_{0}^{2 \pi}\left(\frac{\partial^{2} w}{\partial \varphi^{2}}+w\right)^{2} \mathrm{~d} \varphi
$$

where $E$ is the Young's modulus and $I_{c}=l h^{3} / 12$ is the second moment of area. The radial displacement $w$ of the ring is defined by Equation (1a), and using this equation in 
Equation (6) it can be shown that

$$
U=\frac{E I_{c} \pi}{2 r^{3}}\left[9\left(Q_{1}^{2}+{Q_{2}}^{2}\right)+64\left(R_{1}^{2}+R_{2}^{2}\right)\right]
$$

The strain energy expression accounts for flexural bending of the ring relative to the hub, and Equation (7) indicates that the generalised coordinates of the ring are not coupled in the equation for strain energy.

\subsubsection{Support leg contributions}

Figure 2 shows a ring resonator with 8 uniformly spaced supports. Each support leg is modelled as a thin beam structure connecting the ring to the central hub. The purpose of the legs is to support the ring without significantly influencing the vibration of the ring. Here it is assumed that the legs add negligible mass to the ring, so their contribution to the kinetic energy of the system is considered to be zero.

For simplicity, each support leg is modelled by incorporating radial and tangential springs at the attachment position of the leg to the ring. Denoting the radial and tangential spring stiffness as $k_{r}$ and $k_{t}$, respectively, it can be shown easily that the strain energy for the $j$ 'th support leg located at ring angle $\varphi_{j}$ is given by:

$$
W_{j}=w\left(\varphi_{j}\right)^{2} k_{r} / 2+v\left(\varphi_{j}\right)^{2} k_{t} / 2
$$

where $w\left(\varphi_{j}\right)$ and $v\left(\varphi_{j}\right)$ are the radial and tangential displacements of the ring at the attachment position of the $j$ 'th leg. For 8 uniformly spaced identical legs, the total leg strain energy is given by:

$$
W=\sum_{j=1}^{8} \frac{\left(w\left(\frac{j \pi}{4}\right)\right)^{2}}{2} k_{r}+\sum_{j=1}^{8} \frac{\left(v\left(\frac{j \pi}{4}\right)\right)^{2}}{2} k_{t}
$$

where one of the legs is assumed to be located at angle $\varphi=0$. Using Equations (1a) and (1b) in Equation (9), it can be shown that the total leg strain energy is given by:

$$
\begin{aligned}
W=\frac{k_{r}}{2} & {\left[4 P_{1}^{2}+4{P_{2}}^{2}+4 Q_{1}{ }^{2}+4{Q_{2}}^{2}+4{R_{1}}^{2}+4 R_{2}{ }^{2}\right] \cdots } \\
& +\frac{k_{t}}{2}\left[4\left(P_{1}^{2}+P_{2}^{2}\right)+\left({Q_{1}}^{2}+{Q_{2}}^{2}\right)+\frac{4}{9}\left({R_{1}}^{2}+R_{2}^{2}\right)\right]
\end{aligned}
$$

The number of legs is selected based on the so-called frequency splitting rules $[8,18]$ which ensures each pair of selected generalised coordinates experiences equal stiffness contributions and the frequencies of the perfect resonator remain equal.

\subsection{Rayleigh's dissipation function}

The dominant damping mechanism for millimetre-scale resonators operating in vacuum is thermoelastic damping [32,6,14]. The influence of this and other energy dissipation mechanisms is included in an approximate way via Rayleigh's dissipation function. For the supported ring resonator model considered, Rayleigh's dissipation function [28] can 
be expressed as:

$$
D=\frac{1}{2}\left[c_{P}\left({\dot{P_{1}}}^{2}+{\dot{P_{2}}}^{2}\right)+c_{Q}\left({\dot{Q_{1}}}^{2}+{\dot{Q_{2}}}^{2}\right)+c_{R}\left({\dot{R_{1}}}^{2}+{\dot{R_{2}}}^{2}\right)\right]
$$

where $c_{P}, c_{Q}$, and $c_{R}$ are the viscous damping coefficients for the $1 \theta_{-}, 2 \theta_{-}$, and $3 \theta_{-}$ generalised coordinates, respectively.

\subsection{Electrostatic energies}

The capacitive electrodes shown in Figure 2 are used to apply electrostatic forces to the ring. The electrodes form capacitors with the ring resonator, and for the purposes of analysis the voltages across all outer and inner capacitors are denoted by $V_{o}$ and $V_{i}$, respectively. As the mean radius of the arc capacitors is large compared to the nominal capacitor gap size, over a differential ring angle the capacitors are approximated as parallel plate capacitors. The electrostatic potential energy for a differential element of a parallel plate capacitor is given by [22]

$$
\mathrm{d} E_{\text {cap }}=\frac{\varepsilon_{0} \varepsilon V^{2}}{2 d} \mathrm{~d} A_{c}
$$

where $\varepsilon$ is the relative permittivity, $\varepsilon_{0}$ is the absolute permittivity, $V$ is the electrical potential across the capacitor, $d$ is the gap size, and $\mathrm{d} A_{c}$ is the differential capacitor area. For a constant radius capacitor plate $\mathrm{d} A_{c}=l r_{c} \mathrm{~d} \varphi$, where $r_{c}$ is the mean radius of the capacitor. Figure 5 shows that as the ring vibrates, the gap size $d$ varies around the circumference of the ring, deviating from its nominal gap size $d_{g}$. The magnitude of the deviation depends on the radial deflection $w$ defined in Equation (1a). For the outer capacitors, the gap size is $d_{o}=d_{g}-w$, whilst for the inner capacitors the gap size is $d_{i}=d_{g}+w$.

The electrostatic energy for differential elements for the outer and inner capacitors, respectively, can be expressed as

$$
\begin{aligned}
\mathrm{d} E_{\text {cap }, o} & =\frac{\varepsilon_{0} \varepsilon V_{o}^{2}}{2\left(d_{g}-w\right)} l r_{o} \mathrm{~d} \varphi \\
\mathrm{d} E_{\text {cap }, i} & =\frac{\varepsilon_{0} \varepsilon V_{i}^{2}}{2\left(d_{g}+w\right)} l r_{i} \mathrm{~d} \varphi
\end{aligned}
$$

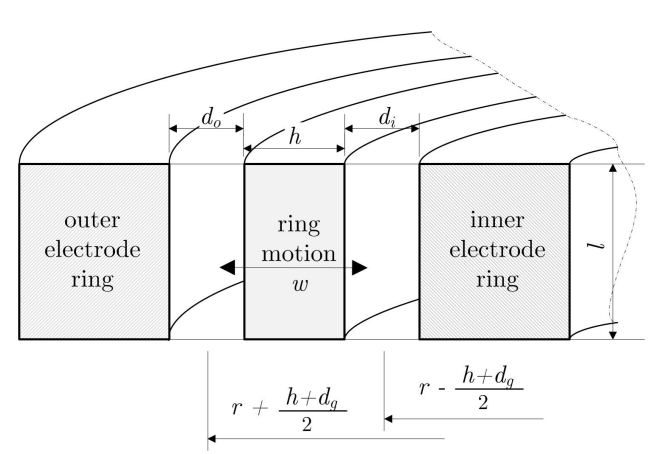

Figure 5: A section of the ring and surrounding plates 
where $r_{o}$ and $r_{i}$ are the mean radii of the outer and inner capacitors. Based on the geometry of the gap, the mean outer and inner radii of the capacitors can be expressed as:

$$
\begin{aligned}
& r_{o}=r+\frac{h+d_{g}}{2} \\
& r_{i}=r-\frac{h+d_{g}}{2}
\end{aligned}
$$

To facilitate the development of analytical expressions to take account of capacitors extending around ring circumference, the gap size terms appearing in Equation (13) are expanded as follows:

$$
\begin{aligned}
& \frac{1}{d_{g}-w}=\frac{1}{d_{g}}\left[1+\frac{w}{d_{g}}+\left(\frac{w}{d_{g}}\right)^{2}+\left(\frac{w}{d_{g}}\right)^{3}+\cdots\right] \\
& \frac{1}{d_{g}+w}=\frac{1}{d_{g}}\left[1-\frac{w}{d_{g}}+\left(\frac{w}{d_{g}}\right)^{2}-\left(\frac{w}{d_{g}}\right)^{3}+-\cdots\right]
\end{aligned}
$$

for the outer and inner capacitors, respectively.

Figure 2 indicates that neighbouring electrodes are separated by circumferential gaps to insulate the electrodes from each other and to allow space for the support legs. These gaps are small compared to the span of the electrodes and are neglected here. This assumption allows the electrodes to be combined together to form one continuous electrode around the complete ring circumference. A detailed study on the significance of insulating gaps between electrodes has been performed in the thesis by Sieberer [24], where it was found that the above assumption yields errors $<10 \%$ for a combined gap span of less than $5 \%$ of the ring circumference. Using Equation (15), the electrostatic potential energies arising from the outer and inner electrodes can be expressed as:

$$
\begin{aligned}
& E_{\mathrm{cap}, o}=\frac{\varepsilon_{0} \varepsilon V_{o}^{2}}{2 d_{g}} \int_{0}^{2 \pi}\left[1+\frac{w}{d_{g}}+\left(\frac{w}{d_{g}}\right)^{2}+\left(\frac{w}{d_{g}}\right)^{3}+\cdots\right] l r_{o} \mathrm{~d} \varphi \\
& E_{\mathrm{cap}, i}=\frac{\varepsilon_{0} \varepsilon V_{i}^{2}}{2 d_{g}} \int_{0}^{2 \pi}\left[1-\frac{w}{d_{g}}+\left(\frac{w}{d_{g}}\right)^{2}-\left(\frac{w}{d_{g}}\right)^{3}+-\cdots\right] l r_{i} \mathrm{~d} \varphi
\end{aligned}
$$

Using Equation (1a) and performing the integration with respect to $\varphi$, expressions for the electrostatic energies for the outer and inner rings of electrodes can be determined. The full derivation of these energies is shown in Appendix A. In what follows the quadratic, cubic and quartic order terms in the generalised coordinates are denoted by $S_{2}, S_{3}$, and $S_{4}$. Higher order terms are not stated for brevity but are included in numerical calculations later. The total electrostatic potential energy $E_{\text {cap }}=E_{\text {cap }, o}+E_{\text {cap }, i}$ is given 
by:

$$
\begin{array}{r}
E_{\text {cap }}=\frac{\varepsilon_{0} \varepsilon l}{2 d_{g}}\left[\left(V_{o}^{2} r_{o}+V_{i}^{2} r_{i}\right) 2 \pi+\left(V_{o}^{2} r_{o}+V_{i}^{2} r_{i}\right) S_{2} \cdots\right. \\
\left.+\left(V_{o}^{2} r_{o}-V_{i}^{2} r_{i}\right) S_{3}+\left(V_{o}^{2} r_{o}+V_{i}^{2} r_{i}\right) S_{4}\right]
\end{array}
$$

where

$$
\begin{aligned}
& S_{2}=\frac{\pi}{d_{g}{ }^{2}}\left[\dot{P}_{1}^{2}+\dot{P}_{2}^{2}+{\dot{Q_{1}}}^{2}+{\dot{Q_{2}}}^{2}+{\dot{R_{1}}}^{2}+{\dot{R_{2}}}^{2}\right] \\
& S_{3}=\frac{3 \pi}{2 d_{g}^{3}}\left[-Q_{1} P_{2}^{2}+Q_{1} P_{1}^{2}+2 Q_{1} R_{1} P_{1} \cdots\right. \\
& \left.+2 Q_{2} P_{1} P_{2}-2 R_{1} Q_{2} P_{2}+2 Q_{1} R_{2} P_{2}+2 Q_{2} R_{2} P_{1}\right] \\
& S_{4}=\frac{\pi}{4 d_{g}{ }^{4}}\left[12 Q_{1}{ }^{2} R_{1}{ }^{2}+3 R_{2}{ }^{4}+12 Q_{2}{ }^{2} R_{2}{ }^{2}+12 Q_{1}{ }^{2} R_{2}{ }^{2}+6 R_{1}{ }^{2} R_{2}{ }^{2} \cdots\right. \\
& +12 Q_{2}{ }^{2} R_{2} P_{2}+3 Q_{1}{ }^{4}+3 R_{1}{ }^{4}+3 Q_{2}{ }^{4}+3 P_{2}{ }^{4}+3 P_{1}^{4}+12 P_{1}{ }^{2} R_{2} P_{2} \ldots \\
& +24 Q_{2} R_{2} Q_{1} P_{1}+24 Q_{1} R_{1} Q_{2} P_{2}-12 R_{1} P_{1} Q_{2}{ }^{2}-12 Q_{1}{ }^{2} R_{2} P_{2} \ldots \\
& -12 R_{1} P_{1} P_{2}^{2}+12 Q_{1}{ }^{2} R_{1} P_{1}+12 R_{1}{ }^{2} Q_{2}{ }^{2}+12 R_{2}{ }^{2} P_{1}{ }^{2}+6 Q_{1}{ }^{2} Q_{2}{ }^{2} \cdots \\
& +12 R_{1}{ }^{2} P_{2}{ }^{2}+12 P_{1}{ }^{2} Q_{2}{ }^{2}+12 R_{2}{ }^{2} P_{2}{ }^{2}-4 P_{2}^{3} R_{2}+12 P_{2}{ }^{2} Q_{2}{ }^{2} \ldots \\
& \left.+12 Q_{1}{ }^{2} P_{2}^{2}+6 P_{1}^{2} P_{2}^{2}+12 R_{1}^{2} P_{1}^{2}+4 P_{1}^{3} R_{1}+12 P_{1}^{2} Q_{1}{ }^{2}\right]
\end{aligned}
$$

The equation for $S_{2}$ indicates that in the linear case the generalised coordinates of the ring are not coupled. However, the equations for $S_{3}$ and $S_{4}$ indicate that the electrostatic nonlinearity couples the generalised coordinates.

\subsection{Governing equations of motion}

The governing equations of motion are derived by substituting the relevant expressions into Equation (2) and performing the required differentiations. It can be shown that the equations of motion can be expressed as:

$$
\begin{aligned}
\ddot{P}_{1}+\frac{\omega_{P}^{*}}{Q_{f, P}} \dot{P}_{1}+\omega_{P}^{* 2} P_{1} & =-\ddot{x}_{b}+\frac{F_{\mathrm{c}, \mathrm{nl}, P_{1}}}{m_{P}} \\
\ddot{P}_{2}+\frac{\omega_{P}^{*}}{Q_{f, P}} \dot{P}_{2}+\omega_{P}^{* 2} P_{2} & =-\ddot{y}_{b}+\frac{F_{\mathrm{c}, \mathrm{nl}, P_{2}}}{m_{P}} \\
\ddot{Q}_{1}+\frac{\omega_{Q}^{*}}{Q_{f, Q}} \dot{Q}_{1}+\omega_{Q}^{* 2} Q_{1} & =\frac{F_{\mathrm{c}, \mathrm{nl}, Q_{1}}}{m_{Q}} \\
\ddot{Q}_{2}+\frac{\omega_{Q}^{*}}{Q_{f, Q}} \dot{Q}_{2}+\omega_{Q}^{* 2} Q_{2} & =\frac{F_{\mathrm{c}, \mathrm{nl}, Q_{2}}}{m_{Q}}
\end{aligned}
$$




$$
\begin{aligned}
& \ddot{R}_{1}+\frac{\omega_{R}^{*}}{Q_{f, R}} \dot{R}_{1}+\omega_{R}^{* 2} R_{1}=\frac{F_{\mathrm{c}, \mathrm{nl}, R_{1}}}{m_{R}} \\
& \ddot{R}_{2}+\frac{\omega_{R}^{*}}{Q_{f, R}} \dot{R}_{2}+\omega_{R}^{* 2} R_{2}=\frac{F_{\mathrm{c}, \mathrm{nl}, R_{2}}}{m_{R}}
\end{aligned}
$$

where the damping is represented by quality factors $Q_{f, N}=m_{N} \omega_{N}^{*} / c_{N}(N=P, Q, R)$, the natural frequencies of the electromechanical resonator, including the influence of electrostatic forces, are given by

$$
\omega_{N}^{*}=\sqrt{\frac{k_{N}-\left(k_{\mathrm{el}, o}+k_{\mathrm{el}, i}\right)}{m_{N}}}
$$

and $F_{\mathrm{c}, \mathrm{nl}, N_{i}} / m_{N}$ are mass-normalised generalised nonlinear electrostatic forces. The modal masses are $m_{P}=m, m_{Q}=5 \mathrm{~m} / 8$, and $m_{R}=10 \mathrm{~m} / 18$ and the modal stiffnesses are $k_{P}=4 k_{r}+4 k_{t}, k_{Q}=4 k_{r}+k_{t}+9 E I_{x} \pi / r^{3}$, and $k_{R}=4 k_{r}+4 k_{t} / 9+64 E I_{x} \pi / r^{3}$.

In these equations the linear electrostatic forces provided by the outer and inner capacitors change the stiffness of the ring by applying a softening effect. These effects are represented by the linear electrostatic stiffness coefficients:

$$
\begin{aligned}
k_{\mathrm{el}, o} & =\frac{\pi \varepsilon_{0} \varepsilon l r_{o} V_{o}^{2}}{d_{g}{ }^{3}} \\
k_{\mathrm{el}, i} & =\frac{\pi \varepsilon_{0} \varepsilon l r_{i} V_{i}^{2}}{d_{g}{ }^{3}}
\end{aligned}
$$

for the outer and inner capacitors respectively and affect the natural frequency equation. The electrostatic forces provide the only source of nonlinearity $\left(F_{\mathrm{c}, \mathrm{nl}}\right)$ and in most practical cases it is valid to neglect these terms because the displacement of the ring (compared to the gap size) is normally small under standard operating conditions. Under these conditions, the electrostatics are linear so there is no coupling between the generalised coordinates, and the pairs of generalised coordinates have equal mass, damping, and stiffness coefficients with the softening effect for all coordinates, increasing as voltages $V_{o}$ and $V_{i}$ are increased.

In this work severe shock conditions are investigated and so it is necessary to include the nonlinear electrostatic force terms as well. As the nonlinearity was expressed in power-series form (see Equation (15)) the level of approximation depends on the number of terms included in Equation (16). The quadratic and cubic nonlinear electrostatic generalised forces $F_{\mathrm{c}, \mathrm{nl}, \mathrm{N}_{\mathrm{i}}}$ (with $\left.\mathrm{nl}=2,3\right)$ are discussed below. 
The quadratic electrostatic forcing terms for the six coordinates are:

$$
\begin{aligned}
& F_{\mathrm{c}, 2, P_{1}}=\frac{3\left(k_{\mathrm{el}, o}-k_{\mathrm{el}, i}\right)}{2 d_{g}}\left(Q_{2} P_{2}+Q_{1} P_{1}+Q_{1} R_{1}+Q_{2} R_{2}\right) \\
& F_{\mathrm{c}, 2, P_{2}}=\frac{3\left(k_{\mathrm{el}, o}-k_{\mathrm{el}, i}\right)}{2 d_{g}}\left(Q_{2} P_{1}-Q_{1} P_{2}+Q_{1} R_{2}-Q_{2} R_{1}\right) \\
& F_{\mathrm{c}, 2, Q_{1}}=\frac{3\left(k_{\mathrm{el}, o}-k_{\mathrm{el}, i}\right)}{4 d_{g}}\left(P_{1}^{2}-P_{2}^{2}+2 P_{1} R_{1}+2 P_{2} R_{2}\right) \\
& F_{\mathrm{c}, 2, Q_{2}}=\frac{3\left(k_{\mathrm{el}, o}-k_{\mathrm{el}, i}\right)}{2 d_{g}}\left(P_{1} P_{2}+P_{1} R_{2}-P_{2} R_{1}\right) \\
& F_{\mathrm{c}, 2, R_{1}}=\frac{3\left(k_{\mathrm{el}, o}-k_{\mathrm{el}, i}\right)}{2 d_{g}}\left(P_{1} Q_{1}-P_{2} Q_{2}\right) \\
& F_{\mathrm{c}, 2, R_{2}}=\frac{3\left(k_{\mathrm{el}, o}-k_{\mathrm{el}, i}\right)}{2 d_{g}}\left(P_{1} Q_{2}+P_{2} Q_{1}\right)
\end{aligned}
$$

These terms are all proportional to $\left(r_{o} V_{\mathrm{o}}^{2}-r_{i} V_{\mathrm{i}}^{2}\right)$, indicating that their magnitude can be reduced by equalising the outer and inner capacitor voltages. These expressions illustrate the presence of quadratic coupling between generalised coordinates $\left(P_{1}, P_{2}, Q_{1}, Q_{2}\right.$, $R_{1}$, and $R_{2}$ ).

Coupling terms involving only one pair of generalised coordinates are referred to here as 'direct coupling'. In Equation (22), direct coupling only occurs for the rigidbody generalised coordinates $\left(P_{1}\right.$ and $\left.P_{2}\right)$, i.e. the terms $P_{1}^{2}$ and $P_{2}^{2}$ in $F_{\mathrm{c}, 2, Q_{1}}$, and the term $P_{1} P_{2}$ in $F_{\mathrm{c}, 2, Q_{2}}$. The resulting forcing is called 'direct forcing', and can exist in higher order terms also. However, no direct coupling exists between the flexural pairs of generalised coordinates in Equation (22). All other coupling terms are referred to here as 'mixed coupling'. These terms include two or more different generalised coordinates and examples of quadratic mixed coupling in Equation (22) include: $Q_{1} P_{1}, Q_{2} R_{2}$. For the rigid-body equations (Equations (22a) and (22b)), the mixed coupling terms include products of $P$ - and $Q$-generalised coordinates, and $R$ - and $Q$-generalised coordinates. These terms induce forcing on the rigid-body coordinates when the relevant $P$ - and $Q$ generalised coordinates or $R$ - and $Q$-generalised coordinates have non-zero displacements. The mixed coupling terms appearing in the $2 \theta$ - and $3 \theta$-equations (Equations (22c) to (22f)) act in a similar way.

The cubic electrostatic forcing terms for the six coordinates are:

$$
\begin{aligned}
& F_{\mathrm{c}, 3, P_{1}}=\frac{3\left(k_{\mathrm{el}, o}+k_{\mathrm{el}, i}\right)}{2 d_{g}{ }^{2}}\left(P_{1}^{3}+\left(P_{1}{ }^{2}-P_{2}{ }^{2}\right) R_{1}+P_{1}\left(2 Q_{1}{ }^{2}+2 Q_{2}{ }^{2}+P_{2}{ }^{2} \cdots\right.\right. \\
& \left.\left.+2 R_{1}{ }^{2}+2 R_{2}{ }^{2}+2 R_{2} P_{2}\right)+2 Q_{1} Q_{2} R_{2}+R_{1} Q_{1}{ }^{2}-R_{1}{Q_{2}}^{2}\right) \\
& F_{\mathrm{c}, 3, P_{2}}=\frac{3\left(k_{\mathrm{el}, o}+k_{\mathrm{el}, i}\right)}{2 d_{g}{ }^{2}}\left(P_{2}{ }^{3}+\left(R_{2}-R_{2}\right) P_{2}{ }^{2}+\left(2 R_{1}{ }^{2}+2 Q_{2}{ }^{2}+P_{1}{ }^{2} \cdots\right.\right. \\
& \left.\left.+2 R_{2}^{2}+2 Q_{1}^{2}-2 R_{1} P_{1}\right) P_{2}+\left(Q_{2}^{2}-Q_{1}^{2}\right) R_{2}+2 Q_{1} R_{1} Q_{2}\right)
\end{aligned}
$$




$$
\begin{aligned}
& F_{\mathrm{c}, 3, Q_{1}}=\frac{3\left(k_{\mathrm{el}, o}+k_{\mathrm{el}, i}\right)}{2 d_{g}{ }^{2}}\left(Q_{1}{ }^{3}+\left({Q_{2}}^{2}+2 P_{1}{ }^{2}+2 R_{1} P_{1}+2 R_{1}{ }^{2}+2 R_{2}{ }^{2} \cdots\right.\right. \\
& \left.\left.+2 P_{2}{ }^{2}-2 R_{2} P_{2}\right) Q_{1}+2 R_{1} Q_{2} P_{2}+2 Q_{2} R_{2} P_{1}\right) \\
& F_{\mathrm{c}, 3, Q_{2}}=\frac{3\left(k_{\mathrm{el}, o}+k_{\mathrm{el}, i}\right)}{2 d_{g}{ }^{2}}\left(Q_{2}{ }^{3}+\left(Q_{1}{ }^{2}+2 R_{1}{ }^{2}+2 P_{1}{ }^{2}+2 P_{2}{ }^{2}+2 R_{2} P_{2} \cdots\right.\right. \\
& \left.\left.+2 R_{2}^{2}-2 R_{1} P_{1}\right) Q_{2}+2 Q_{1} R_{1} P_{2}+2 R_{2} Q_{1} P_{1}\right) \\
& F_{\mathrm{c}, 3, R_{1}}=\frac{\left(k_{\mathrm{el}, o}+k_{\mathrm{el}, i}\right)}{2 d_{g}{ }^{2}}\left(3 R_{1}{ }^{3}+\left(6 P_{2}{ }^{2}+6 Q_{2}{ }^{2}+6 P_{1}{ }^{2}+6 Q_{1}{ }^{2}+3 R_{2}{ }^{2}\right) R_{1} \cdots\right. \\
& \left.+P_{1}^{3}-3 P_{1} P_{2}^{2}-3 P_{1} Q_{2}^{2}+3 P_{1} Q_{1}^{2}+6 Q_{1} Q_{2} P_{2}\right) \\
& F_{\mathrm{c}, 3, R_{2}}=\frac{\left(k_{\mathrm{el}, o}+k_{\mathrm{el}, i}\right)}{2 d_{g}{ }^{2}}\left(3 R_{2}{ }^{3}+\left(3 R_{1}{ }^{2}+6 Q_{2}{ }^{2}+6 P_{1}^{2}+6 Q_{1}{ }^{2}+6 P_{2}^{2}\right) R_{2} \cdots\right. \\
& \left.-P_{2}{ }^{3}+3 P_{1}^{2} P_{2}-3 Q_{1}{ }^{2} P_{2}+6 Q_{2} Q_{1} P_{1}+3 P_{2} Q_{2}{ }^{2}\right)
\end{aligned}
$$

These terms are all proportional to $\left(r_{o} V_{\mathrm{o}}^{2}+r_{i} V_{\mathrm{i}}^{2}\right)$, indicating that the magnitude can only be diminished by simultaneously reducing the outer and inner capacitor voltages, and illustrate the presence of 'modal softening' terms, direct coupling and mixed coupling. Cubic modal softening occurs in all cases (Equations (23a) to (23f)), when the equation of motion for a particular generalised coordinate contains a pure cubic term in the same generalised coordinate, indicating nonlinear behaviour of the generalised coordinate for large modal displacements. The only direct forcing occurring in these equations is in Equations (23e) and (23f). These equations govern the $3 \theta$-response but are directly influenced by the $1 \theta$-motion through the $\left(P_{1}^{3}-3 P_{1} P_{2}{ }^{2}\right)$ and $\left(-P_{2}{ }^{3}+3 P_{1}{ }^{2} P_{2}\right)$ terms. All other couplings involve mixed coupling terms.

Quartic and higher order nonlinear forcing terms can be obtained by including higher order terms in the power series in the electrostatic energy expression in Equation (16). It can be shown that all even order forcing terms (e.g. quartic order) are proportional to $\left(r_{o} V_{\mathrm{o}}^{2}-r_{i} V_{\mathrm{i}}^{2}\right)$ and all odd order forcing terms (e.g. quintic order) are proportional to $\left(r_{o} V_{\mathrm{o}}^{2}+r_{i} V_{\mathrm{i}}^{2}\right)$. Furthermore, direct forcing of $1 \theta$-motion to flexural response is given by direct coupling terms in the equations governing the $2 \theta$-response for even higher order terms, and in the equations governing the $3 \theta$-response for odd higher order terms. The significance of higher order terms for a specific shock situation is investigated in Section 3.5.

\section{Numerical simulations}

In this section, the influence of the nonlinear electrostatic coupling on the response of the $2 \theta$ - and $3 \theta$-generalised coordinates is determined for a specific sensor design. By calculating the time histories and the spectral content of these responses, the dominant coupling mechanisms are identified and quantified. The equations of motion derived in Section 2 are used to calculate the shock response of the system to an applied half- 
sine shock pulse with zero initial conditions. An 'ode23' numerical integrator is used to generate the simulation results and the spectral density of the time-responses is obtained as the squared value of the FFT-transformation. For all simulation results presented, the ring properties reported in Table 2 are used. These geometrical properties and natural frequencies are based on the device described in [33] and the quality factors used are assumed high for a resonator operating in an ultra-low pressure atmosphere.

\begin{tabular}{ccccc} 
Ring property & Symbol & Value & \\
\hline Material: & Silicon $\operatorname{Si}(111)$ & & \\
Mean radius & $r$ & $1.5 \times 10^{-3} \mathrm{~m}$ & & \\
Axial length & $l$ & $1.5 \times 10^{-4} \mathrm{~m}$ & & \\
Radial thickness & $h$ & $1.8 \times 10^{-4} \mathrm{~m}$ & & \\
Nominal gap size & $d_{g}$ & $1.0 \times 10^{-5} \mathrm{~m}$ & & \\
Capacitor voltage & $V_{o}$ & $50 \mathrm{~V}$ & & \\
\hline Ring motions & Linear natural frequencies & $Q_{f}$-factors \\
$1 \theta$-motion & $f_{P}$ & $10900 \mathrm{~Hz}$ & $Q_{f, P}$ & 30000 \\
$2 \theta$-motion & $f_{Q}$ & $17300 \mathrm{~Hz}$ & $Q_{f, Q}$ & 60000 \\
$3 \theta$-motion & $f_{R}$ & $22100 \mathrm{~Hz}$ & $Q_{f, R}$ & 60000 \\
\hline
\end{tabular}

Table 2: Properties for the ring resonator

The section is organised as follows. The applied shock is defined in Section 3.1. The simulations presented in Sections 3.2 and 3.3 consider quadratic and cubic electrostatic forces only and identify the dominant coupling mechanisms in the resonator. Results comparing the cases when the outer electrodes only and the outer and inner electrodes are activated are presented and discussed in Section 3.4. This is followed in Section 3.5 by a convergence study in which the significance of higher order electrostatic terms on the response is studied for the shock input considered. Section 3.6 considers the maximum shock induced flexural response and converts it to an applied rate input to assess the significance of the coupling to sensor applications. Finally, Section 3.7 describes a parameter study to investigate the influence of the ratio of the $2 \theta / 1 \theta$ - and $3 \theta / 1 \theta$ frequencies on the coupling and the ratio that minimises the nonlinear coupling.

\subsection{Shock application}

The shock is applied in the $x$-direction (see Figure 4) as a half-sine acceleration pulse, i.e.

$$
\begin{aligned}
& \ddot{x}=\left\{\begin{array}{cc}
A_{s} \sin \left(\frac{\pi t}{T_{s}}\right) & \text { for } t \leq T_{s} \\
0 & \text { for } t>T_{s}
\end{array}\right. \\
& \ddot{y}=0
\end{aligned}
$$

where $A_{s}$ is the shock amplitude and $T_{s}$ is the shock duration. This form of shock can be used to represent excitations applied in pendulum tests $[27,25]$ and is used in high $g$ shock testing. The shock response is characterised as being quasi-static or dynamic and is governed by the shock duration. Figure 6 shows the rigid body shock response spectrum obtained by determining the maximum rigid body response for different shock 
durations using the developed model. Sieberer [25] found that the dynamic rigid-body responses include significant mode coupling and a practical shock duration yielding dynamic rigid-body shock responses is investigated here. Dynamic shock responses occur when the shock duration is in the range of half of the natural period of vibration of the rigid-body motion. A half-sine base excitation with shock amplitude $A_{s}=16000 \mathrm{~m} / \mathrm{s}^{2}$ and shock duration $T_{s}=0.1 \mathrm{~ms}$ is used. For the ring considered, half the time period for the rigid-body frequency neglecting electrostatic softening is $0.046 \mathrm{~ms}$.

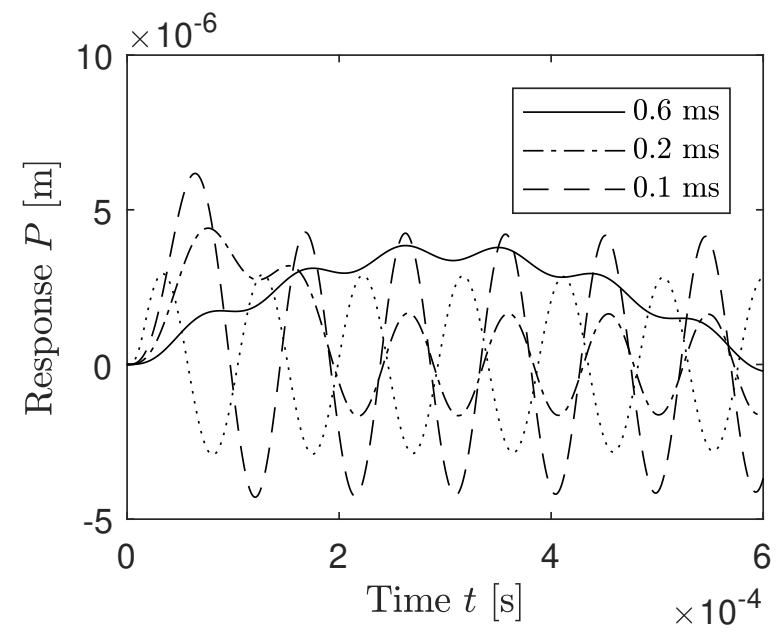

(a) Time response

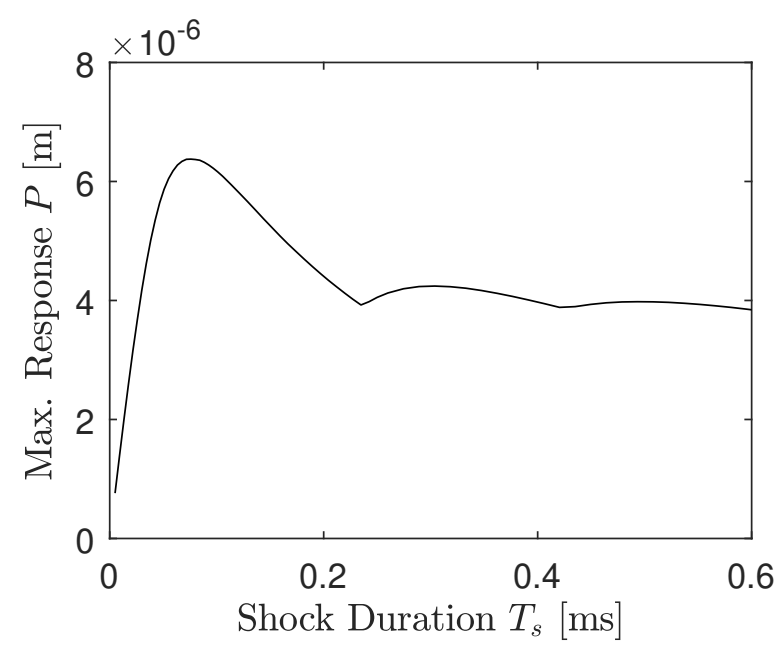

(b) Shock response spectrum

Figure 6: Time responses to different shocks and resulting shock response spectrum of the maximum response. In (a), the solid line is a quasi-static response, and the dashed and dotted lines are dynamic responses. The diagram in (b) shows the spectrum of maximum shock responses.

It has been reported by Harris [13] that different shock pulses with finite slope, e.g. haversine or triangular pulses, exhibit a qualitatively similar response characteristic. This means that for such shock pulses, the only significant difference in the rigid-body response is expected to be a different maximum response amplitude. A rectangular acceleration shock pulse yields dynamic response regardless of the shock duration, as the whole frequency spectrum is excited. However, the residual vibration amplitude is not generally large and can theoretically be zero in the case of the shock duration equalling the natural period of the excited mode of vibration.

The applied shock does not excite the $P_{2}$-generalised coordinate directly and the structure of the nonlinear coupling terms in Equations (22) and (23) ensures there is no coupling between the shock-excited $P_{1}$-generalised coordinate and the $P_{2}$-generalised coordinate.

The following sections present numerical simulation results for the shock response and quantify the responses of the generalised coordinates.

\subsection{Simulations with outer electrode activated only}

In this section, the influence of the nonlinear electrostatic coupling on the response of the $Q_{1^{-}}$and $R_{1}$-generalised coordinates is determined for the design specified in Table 2 with inactive inner electrodes (i.e. outer electrodes activated only). This is achieved by 
choosing the inner ring of electrodes to have the same potential as the ring which ensures the inner capacitor voltage is zero.

Figures 7, 8, and 9 show the rigid-body and flexural responses obtained for each of the generalised coordinates, and include time histories and power spectral densities of the responses. These plots are used to help identify the dominant coupling terms.

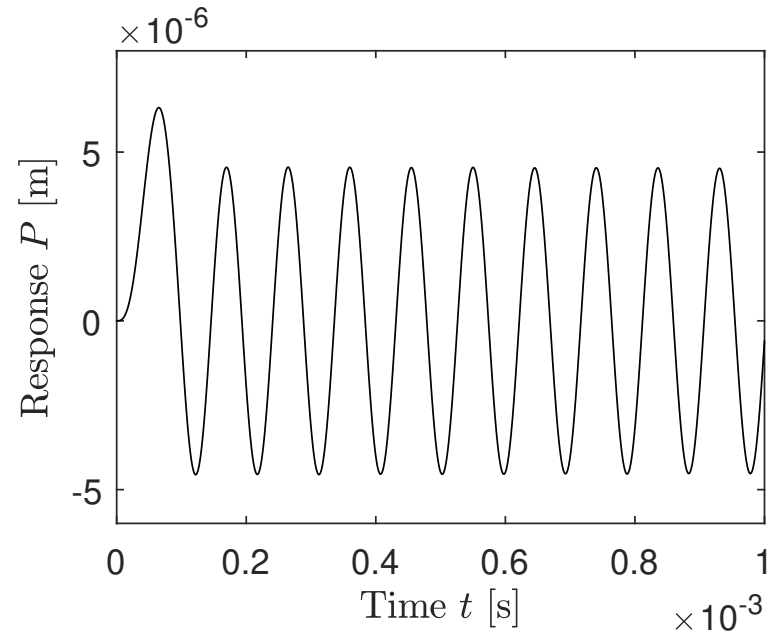

(a) Time response

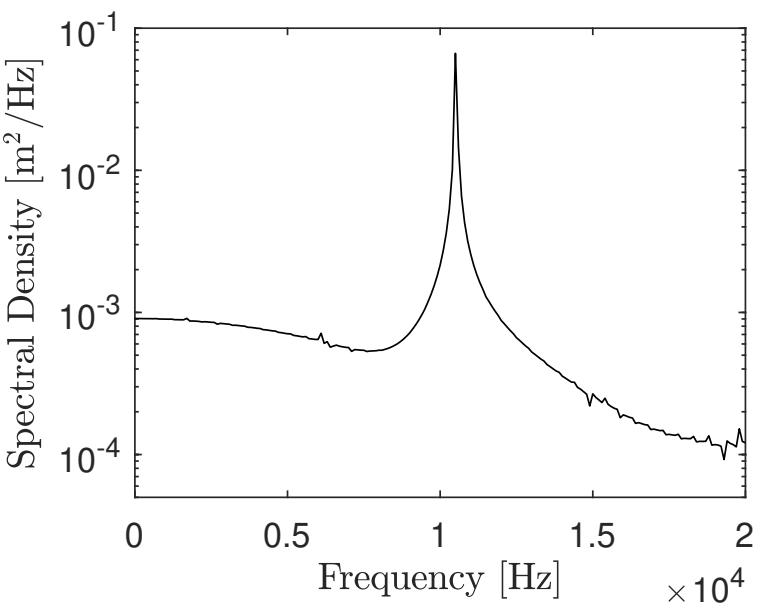

(b) FFT response

Figure 7: Time domain and frequency domain shock response of the rigid-body motion in the $P_{1}$ coordinate. The natural frequency for the rigid-body modes is given in Table 2 .

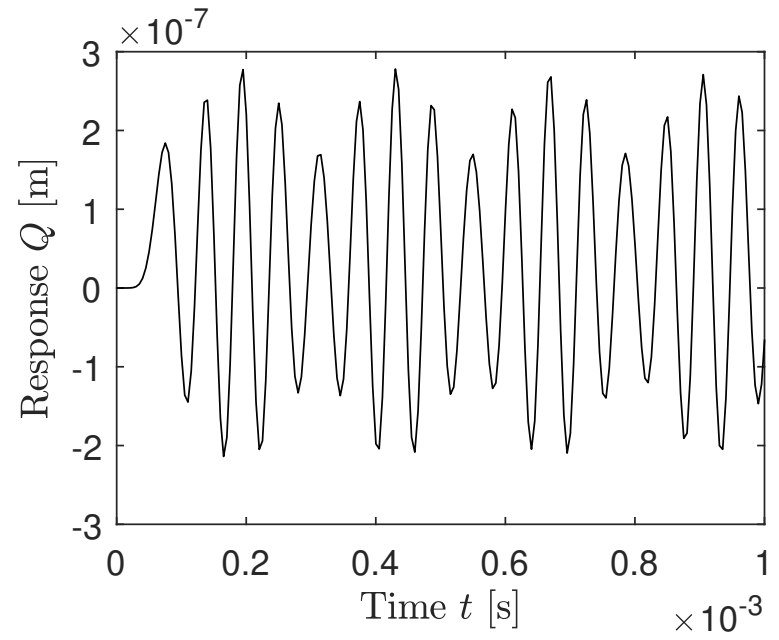

(a) Time response

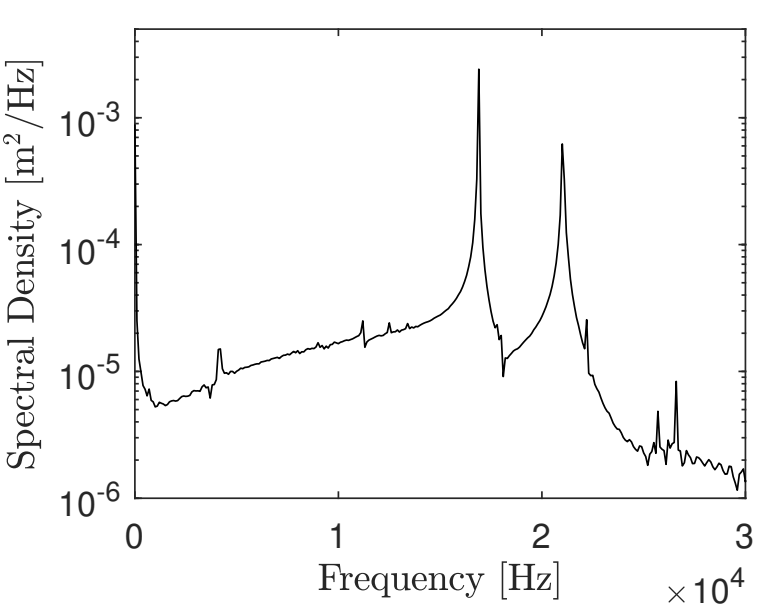

(b) FFT response

Figure 8: Time domain and frequency domain shock response of the motion in the $Q_{1}$-coordinate. The natural frequency for the $2 \theta$-modes is given in Table 2.

Figure 7 indicates that the $P_{1}$-generalised coordinate oscillates at the rigid body $f_{P}$ frequency $(10.9 \mathrm{kHz})$ and decays slowly because of the low levels of damping present in the sensor. 


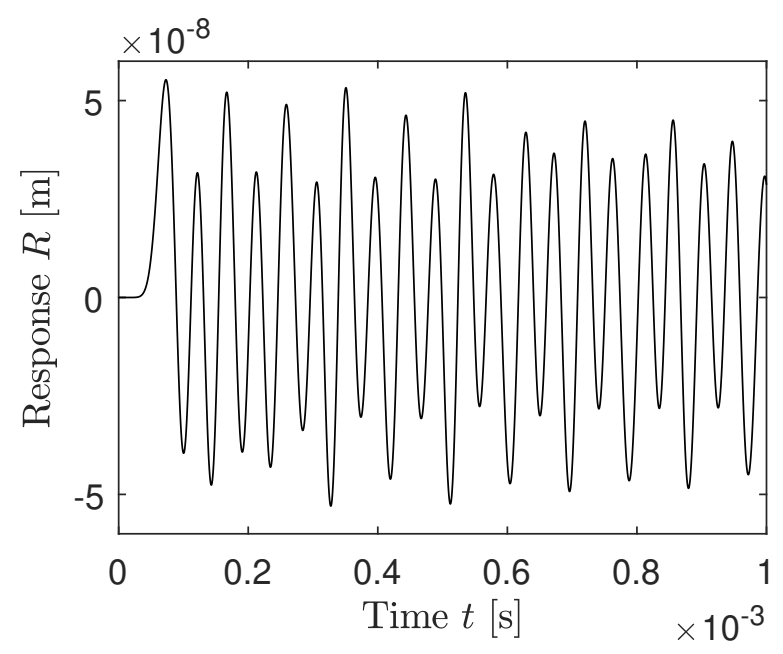

(a) Time response

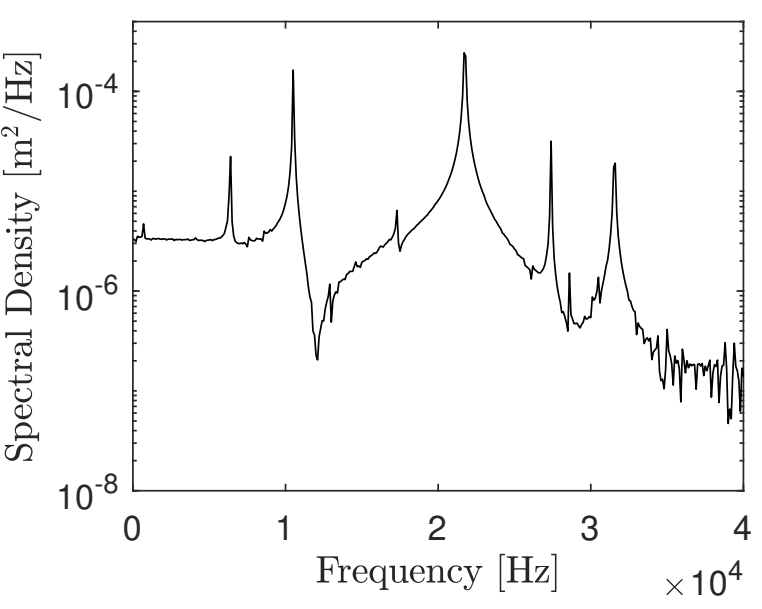

(b) FFT response

Figure 9: Time domain and frequency domain shock response of the motion in the $R_{1}$-coordinate. The natural frequency for the $3 \theta$-modes is given in Table 2.

Figure 8 indicates that the $Q_{1}$ response is much smaller than the $P_{1}$ response and contains frequency components at the $f_{Q}$-frequency $(16.9 \mathrm{kHz})$ and twice the $f_{P}$ frequency $(2 \mathrm{x} 10.9=21.8 \mathrm{kHz})$. The $2 f_{P}$ component arises because the $P_{1}^{2}$ direct coupling terms in Equation (22c) produce frequency content at twice the rigid-body frequency because $\sin ^{2} \omega_{P}=0.5\left(1-\cos 2 \omega_{P}\right)$, which also explains the presence of the static term in the spectrum.

Figure 9 indicates that the $R_{1}$ response is much smaller than the $Q_{1}$ response and contains frequency components at $f_{R}(21.8 \mathrm{kHz}), f_{P}(10.9 \dot{\mathrm{kHz}}), 3 f_{P}(3 \mathrm{x} 10.9=32.7 \mathrm{k} \mathrm{Hz})$ and $f_{P}+f_{Q}(10.9+16.9=27.8 \mathrm{kHz})$. The $3 f_{P}$ component arises because the $P_{1}{ }^{3}$ direct coupling terms in Equation (23e) produce frequency content at three times the rigid-body frequency because $\sin ^{3} \omega_{P}=0.25\left(3 \sin \omega_{P}-\sin 3 \omega_{P}\right)$, which also explains the presence of $f_{P}$. The $f_{P}+f_{Q}$ component arises from the $P_{1} Q_{1}$ mixed coupling term in Equation (22e).

These results have shown that severe shocks can induce coupled responses in the $Q_{1}$ and $R_{1}$ generalised coordinates and have identified the dominant coupling mechanisms for the $Q_{1}$ and $R_{1}$-generalised coordinates. In what follows, the influence of these responses on rate measurements is considered.

\subsection{Frequency filtering of the flexural response}

In ring-based CVGs the angular rate is detected by measuring the response of the sense mode at its resonant frequency. However, as severe shocks can induce responses in the sense modes, these responses will be interpreted by the sensor as angular rate, when angular rate is not applied. The sense electrodes can be arranged to detect the sense mode response only, so the shock-induced erroneous rate output can be determined for a device operating using $2 \theta$-modes by analysing the shock induced response in the $2 \theta$-mode at the $2 \theta$-frequency. As this shock induced response includes a multitude of coupled responses it is necessary to filter these responses to calculate the response at the $2 \theta$-mode frequency. In this work a $6^{\text {th }}$ order elliptic band-pass filter is used to calculate the amplitude of the $2 \theta$-mode frequency over a frequency range centred on the natural frequencies. The 
elliptic filter is chosen because of the sharp cut-off, which enables the filtering of close frequencies, and despite the ripple which is present over the whole frequency range [17]. A similar approach can be used to evaluate a device operating using $3 \theta$-modes, and the filter properties selected for devices operating using $2 \theta$ - and $3 \theta$-modes are summarised in Table 3 , where 'ripple in bandpass' describes the fluctuation maxima in the transmissibility in the pass-band, and 'stop-band $\mathrm{dB}$ down' indicates the reduction in transmissibility in the stop-band. With this filter, the responses at the relevant frequencies are obtained.

\begin{tabular}{ccc} 
Parameter & $2 \theta$-filter & $3 \theta$-filter \\
\hline Lower cut-off frequency & $15000 \mathrm{~Hz}$ & $20000 \mathrm{~Hz}$ \\
Upper cut-off frequency & $20000 \mathrm{~Hz}$ & $25000 \mathrm{~Hz}$ \\
Ripple in bandpass & $0.5 \mathrm{~dB}$ & $0.5 \mathrm{~dB}$ \\
Stop-band dB down & $30 \mathrm{~dB}$ & $30 \mathrm{~dB}$ \\
\hline
\end{tabular}

Table 3: Parameters used in the filter design.

Figure 10 compares the time histories obtained in Figures 8 and 9 with those obtained by filtering the responses.

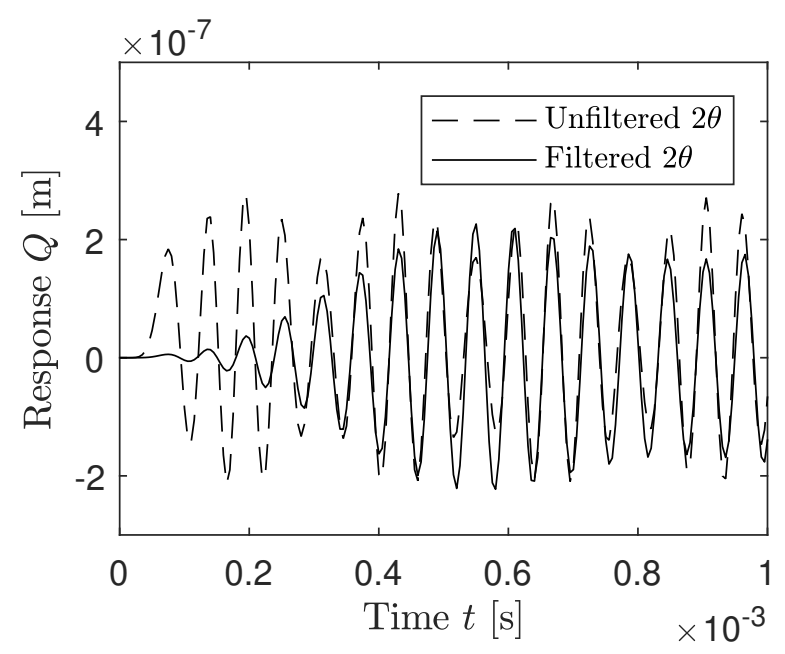

(a) $2 \theta$-response

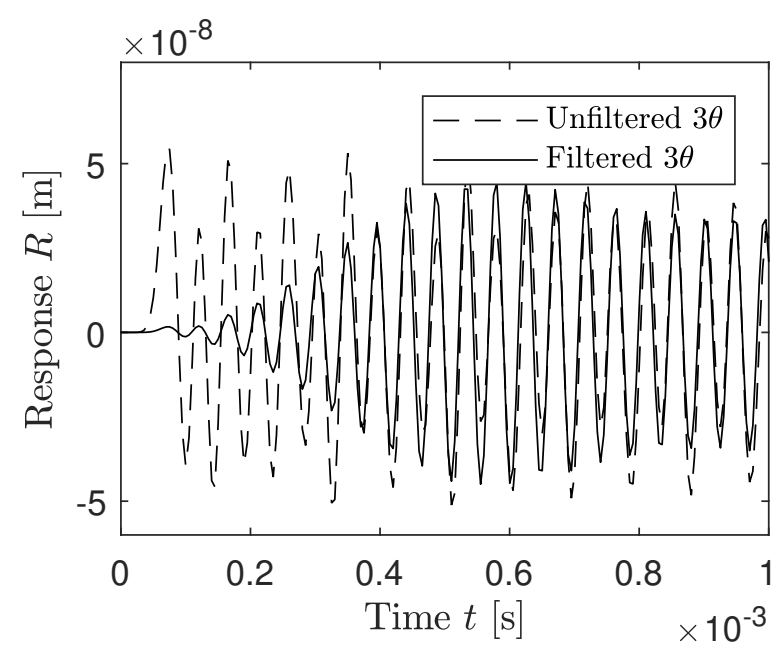

(b) $3 \theta$-response

Figure 10: Time histories of the filtered responses to shock. The solid lines are the responses at the natural frequency of the respective modes, and the dashed lines the unfiltered responses.

The filtered response, i.e. the response at the $2 \theta$-frequency in the $2 \theta$-response, is $0.209 \mu \mathrm{m}$ and is $25 \%$ smaller than the unfiltered $2 \theta$-response, which includes response at the $2 f_{P}$-frequency and other frequencies. The amplitude of vibration at the $3 \theta$-frequency in the $3 \theta$-response is $45.0 \mathrm{~nm}$, and is $15 \%$ smaller than the unfiltered $3 \theta$-response. As such the response used in the rate detection process is smaller than the modal response amplitude, and indicates that the modal response alone does not provide information about the expected influence on the rate measurement. The filtered responses are used later to calculate the equivalent shock induced rate output for devices operating using $2 \theta$ - and $3 \theta$-modes. 


\subsection{Inner electrode activation}

Some ring rate sensors feature inner and outer electrodes to counteract the effects of imperfections by applying different voltages to the inner capacitors [11]. For a perfect ring resonator the capacitor voltage across all inner capacitors is equal. Equations (22) indicate that the quadratic coupling terms are proportional to $\left(r_{o} V_{\mathrm{o}}^{2}-r_{i} V_{\mathrm{i}}^{2}\right)$. These quadratic terms (and all even-ordered terms) can be reduced by ensuring this term is small. For example. if $V_{\mathrm{i}}=V_{\mathrm{o}}$ this can be achieved by selecting $\left(r_{o}-r_{i}\right)$ to be small. Conversely, Equations (23) show that the cubic coupling terms depend on $\left(r_{o} V_{\mathrm{o}}^{2}+r_{i} V_{\mathrm{i}}^{2}\right)$, and so the magnitude of the cubic (and all higher odd-ordered coupling terms) increase as the quadratic terms decrease. In practice this can be achieved by increasing $V_{i}$ from zero, which modifies the linear electrostatic stiffness and the nonlinear coupling terms: the coupling to the $2 \theta$-coordinate reduces as it is governed by quadratic terms, while the coupling to the $3 \theta$-coordinate increases as it is governed by cubic terms.

\begin{tabular}{ccrrr}
\hline$V_{i}$ & $\begin{array}{c}\text { Line-style } \\
\text { (Figs. 11a, 11b) }\end{array}$ & $\begin{array}{r}\text { Rel. Shock } \\
\text { Response } P_{1}\end{array}$ & $\begin{array}{r}\text { Rel. Shock } \\
\text { Response } Q_{1}\end{array}$ & $\begin{array}{r}\text { Rel. Shock } \\
\text { Response } R_{1}\end{array}$ \\
\hline $50 \mathrm{~V}$ & solid & $110 \%$ & $3 \%$ & $260 \%$ \\
$40 \mathrm{~V}$ & dashed & $106 \%$ & $46 \%$ & $187 \%$ \\
$30 \mathrm{~V}$ & dotted & $103 \%$ & $74 \%$ & $142 \%$ \\
$0 \mathrm{~V}$ & dash-dotted & $100 \%$ & $100 \%$ & $100 \%$ \\
\hline
\end{tabular}

Table 4: Resulting coupled response of the rigid-body and flexural motions on gradual increase of the inner capacitor voltage.

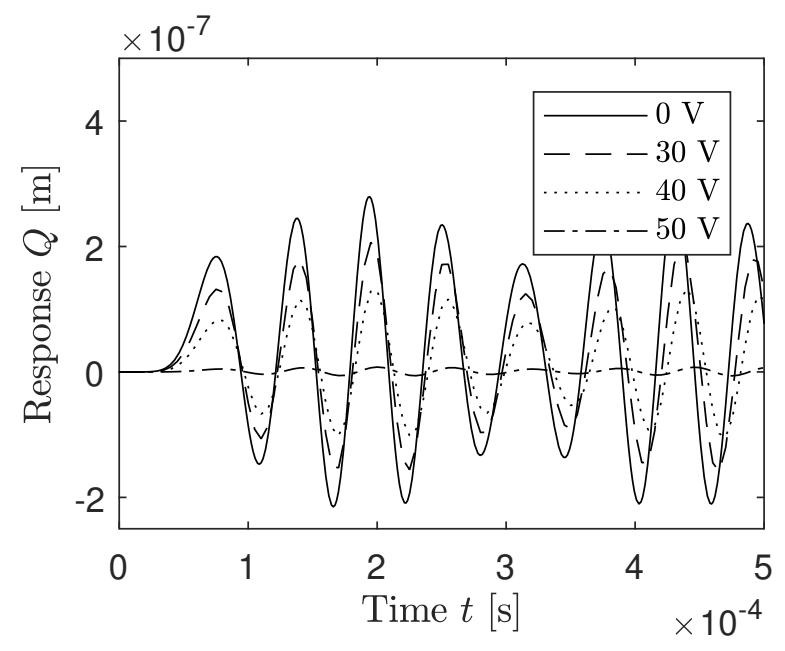

(a) $Q_{1}$-response

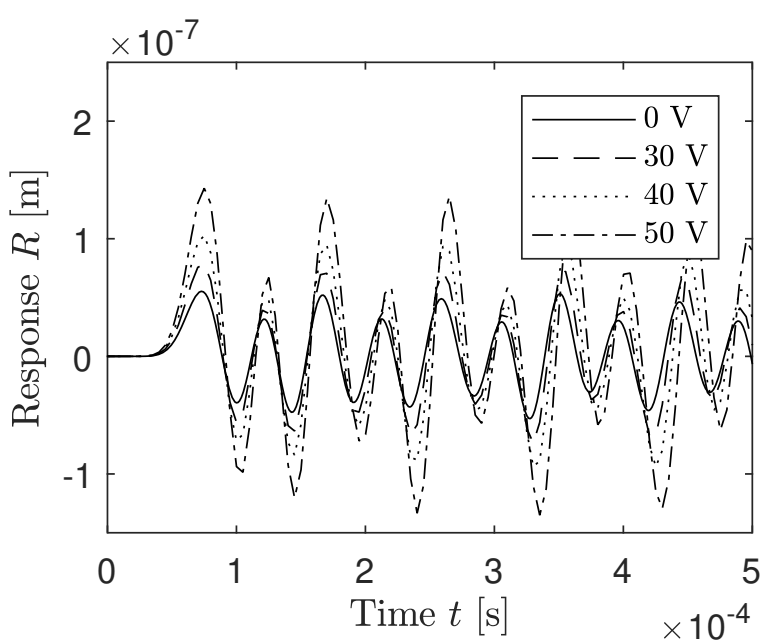

(b) $R_{1}$-response

Figure 11: Flexural $Q_{1}$ - and $R_{1}$-responses on gradual inner electrode activation. The response is reduced with increasing $V_{i}$. A legend is given in Table 4.

Figure 11 illustrates the change in flexural response as the voltage applied to the inner electrodes is increased gradually from 0 to $50 \mathrm{~V}$. Table 4 reports the values of $V_{i}$ 
used in simulations together with the corresponding line styles used in Figure 11, and reports the relative change in modal responses. Figure 11a indicates the reduction in coupling that can be achieved for the $2 \theta$-generalised coordinate, where the coupled $2 \theta$ response diminishes to $8 \mathrm{~nm}$. Figure $11 \mathrm{~b}$ shows that the $3 \theta$-generalised coordinate more than doubles when $V_{o}=V_{i}$, where the coupled $3 \theta$-response increases to $143 \mathrm{~nm}$. These results indicate that having an inner ring of electrodes with non-zero voltage across the inner capacitors can reduce the coupling to the $2 \theta$-generalised coordinates. In contrast, the response of the $3 \theta$-generalised coordinates increases. In addition it is worth noting that the frequencies all reduce as the voltage increases due to electrostatic softening, and the separation of $3 \theta$ - and $1 \theta$-frequencies does not affect direct coupling to the $3 \theta$-response.

\subsection{Higher order nonlinear terms}

The results considered in previous sections are limited to quadratic and cubic electrostatic generalised forcing functions $((22)$ and $(23))$ to illustrate and identify the dominant coupling mechanisms. In the presence of severe shocks and large gap size variations, higher order electrostatic terms can become important. In this section, higher order nonlinear forcing terms up to $9^{\text {th }}$ order are included in the force expressions $F_{\mathrm{c}, \mathrm{nl}, N_{i}}$ and a convergence study is performed with the sensor and shock parameters used earlier to assess the influence of higher order terms on the response.

As discussed at the end of Section 2.4, higher order coupling terms can be obtained in a similar way to the quadratic and cubic coupling terms and included in the nonlinear generalised forcing functions on the right hand side of the equations of motion. As higher order terms are included, the nonlinear forcing terms become increasingly complicated due to the increasingly large number of coupling terms. However, noting that the results presented in Section 3.2 indicated that the coupling between the $1 \theta$-motion and the $2 \theta$ and $3 \theta$-responses is dominated by direct-forcing terms, and a shock applied to the $P_{1^{-}}$

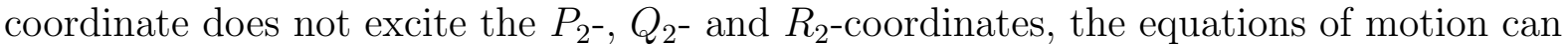
be simplified by only including direct-forcing terms in the equations of motion for the

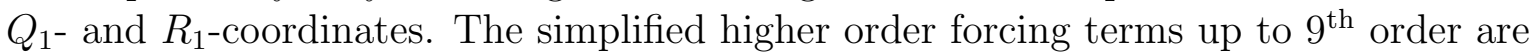

$$
\begin{aligned}
F_{\mathrm{c}, 4, \mathrm{df}, Q_{1}} & =\frac{10 \pi \varepsilon_{0} \varepsilon l}{8 d_{g}{ }^{6}}\left(r_{o} V_{o}^{2}-r_{i} V_{i}^{2}\right) P^{4} \\
F_{\mathrm{c}, 5, \mathrm{df}, R_{1}} & =\frac{15 \pi \varepsilon_{0} \varepsilon l}{16 d_{g}{ }^{7}}\left(r_{o} V_{o}^{2}+r_{i} V_{i}^{2}\right) P^{5} \\
F_{\mathrm{c}, 6, \mathrm{df}, Q_{1}} & =\frac{105 \pi \varepsilon_{0} \varepsilon l}{64 d_{g}{ }^{8}}\left(r_{o} V_{o}^{2}-r_{i} V_{i}^{2}\right) P^{6} \\
F_{\mathrm{c}, 7, \mathrm{df}, R_{1}} & =\frac{21 \pi \varepsilon_{0} \varepsilon l}{16 d_{g}{ }^{9}}\left(r_{o} V_{o}^{2}+r_{i} V_{i}^{2}\right) P^{7} \\
F_{\mathrm{c}, 8, \mathrm{df}, Q_{1}} & =\frac{63 \pi \varepsilon_{0} \varepsilon l}{32 d_{g}{ }^{10}}\left(r_{o} V_{o}^{2}-r_{i} V_{i}^{2} P^{8}\right. \\
F_{\mathrm{c}, 9, \mathrm{df}, R_{1}} & =\frac{315 \pi \varepsilon_{0} \varepsilon l}{192 d_{g}{ }^{11}}\left(r_{o} V_{o}^{2}+r_{i} V_{i}^{2}\right) P^{9}
\end{aligned}
$$

Figure 12 shows how the $Q_{1^{-}}$and $R_{1}$-responses change as higher order terms are included in the electrostatic forcing functions, with the inner electrodes de-activated (i.e. $V_{i}=0$ ). 
The coupled responses increase as higher order terms are included and a relatively good approximation is achieved using a $5^{\text {th }}$ order forcing function. Converged responses are achieved using $9^{\text {th }}$ order forcing functions and for this case the response maxima are $0.552 \mu \mathrm{m}$ in the $2 \theta$-coordinate and $0.120 \mu \mathrm{m}$ in the $3 \theta$-coordinate.

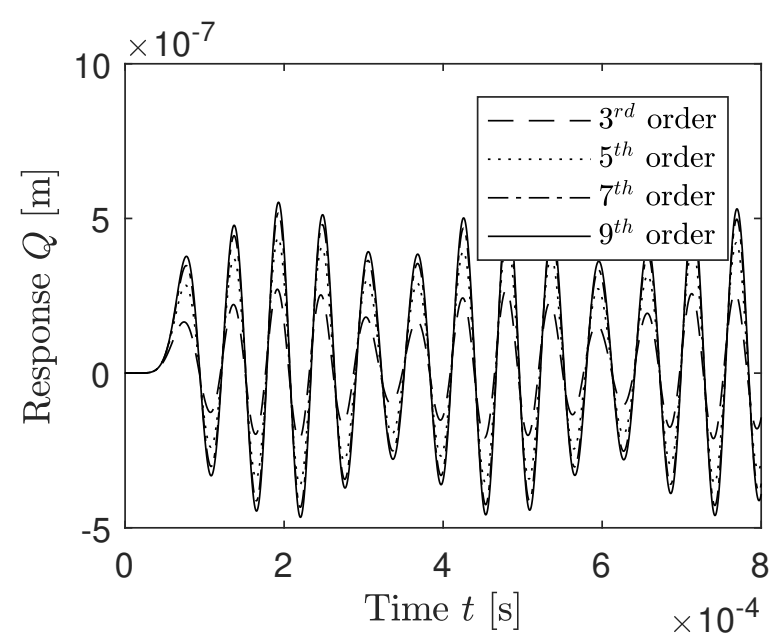

(a) $2 \theta$-response

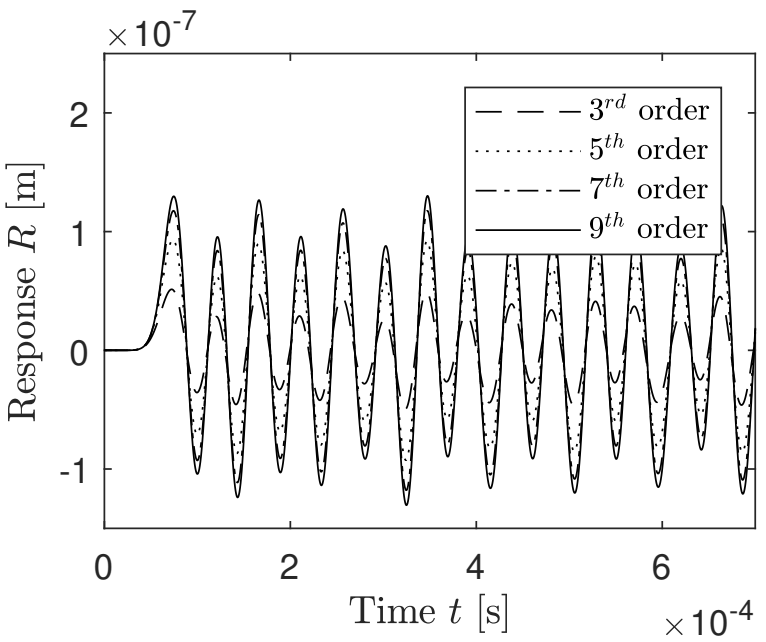

(b) $3 \theta$-response

Figure 12: Flexural responses with higher order electrostatic coupling and inner electrodes deactivated. Dashed, solid, dash-dotted, and dotted lines represent cubic, quintic, $7^{\text {th }}$ order, and $9^{\text {th }}$ order forcing function, respectively.

Figure 13 shows how the $Q_{1^{-}}$and $R_{1^{-}}$-responses change as higher order terms are included in the electrostatic forcing functions, with the inner voltage activated $V_{i}=V_{o}$. Similar to Figure 12, the coupled responses increase as higher order terms are included and $5^{\text {th }}$ order forcing functions yield good approximations for the $2 \theta$ - and $3 \theta$-generalised coordinates. Convergence is also reached for the $9^{\text {th }}$ order forcing function, and the maximum obtained $2 \theta$ - and $3 \theta$-responses are $26 \mathrm{~nm}$ and $508 \mathrm{~nm}$, respectively when the inner electrode is activated.

To determine the response used in the rate detection process and obtain the equivalent rate output, the filtered responses are calculated using the filters described in Table 3 . The filtered $2 \theta$ - and $3 \theta$-responses are shown in Figure 14 . The maximum filtered $2 \theta$-response at the $2 \theta$-frequency is $535 \mathrm{~nm}$, and the maximum filtered response in the $3 \theta$-response at the $3 \theta$-frequency is $168 \mathrm{~nm}$. When the inner electrodes are activated, the filtered $2 \theta$ response reduces to $30 \mathrm{~nm}$ indicating a significant improvement in performance. The $3 \theta$-response increases to $563 \mathrm{~nm}$, yielding reduced performance. The influence of these responses on measured applied rate is considered next.

\subsection{Comparison to an applied rate}

When operating as a rate sensor, the response of the $2 \theta$-companion mode is proportional to the applied angular rate. It was shown in Sections 3.2 and 3.5 that severe shock excitation can induce $2 \theta$-responses, and these responses can be misinterpreted as having arisen from angular rate. In this section, the $2 \theta$-responses arising from severe shocks are 


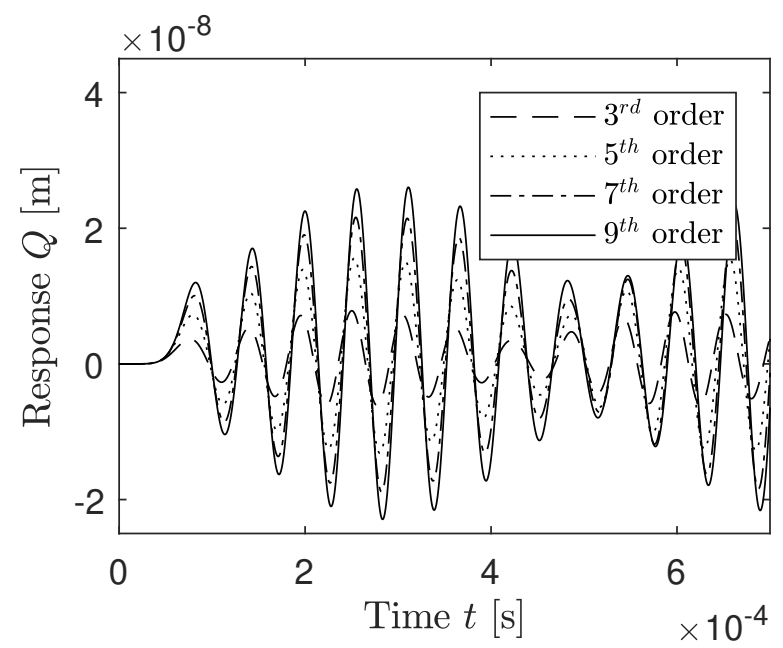

(a) $2 \theta$-response

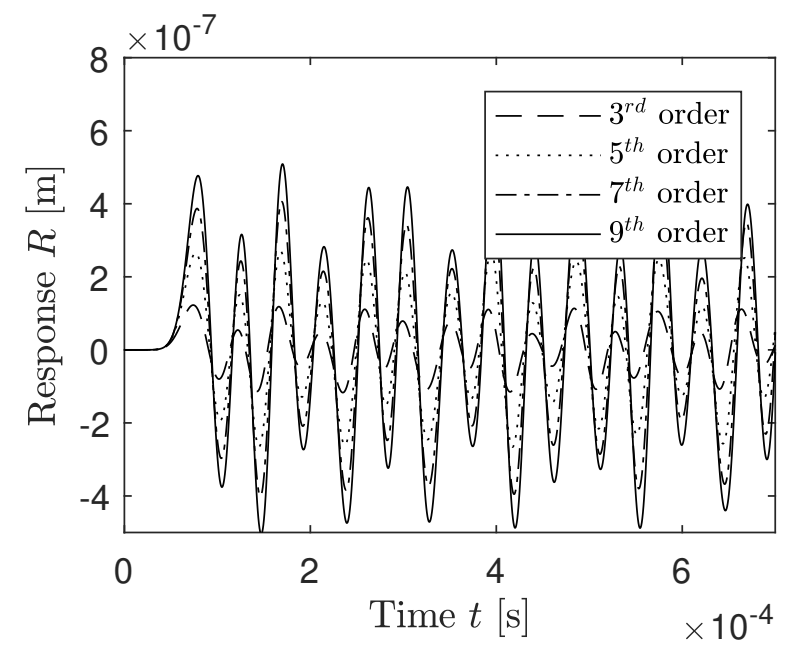

(b) $3 \theta$-response

Figure 13: Flexural responses with higher order electrostatic coupling and inner electrodes activated with $V_{i}=V_{o}$. Dashed, solid, dash-dotted, and dotted lines represent cubic, quintic, $7^{\text {th }}$ order, and $9^{\text {th }}$ order forcing function, respectively.

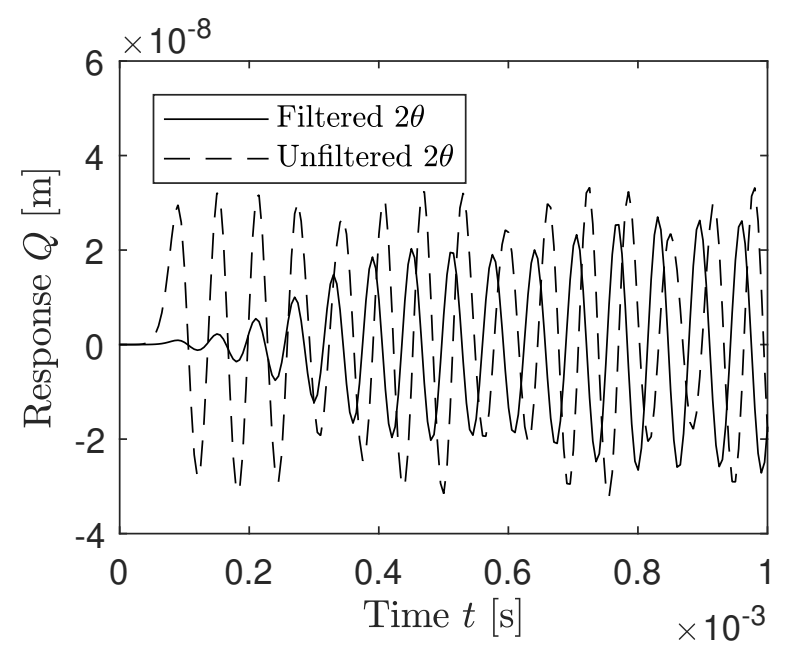

(a) $2 \theta$-response

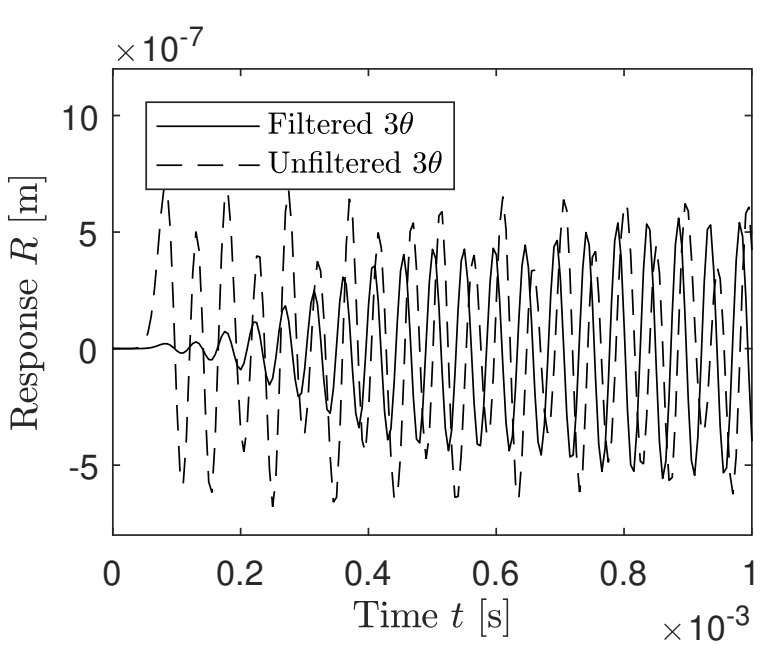

(b) $3 \theta$-response

Figure 14: Time histories of the filtered responses to shock including higher order nonlinear forces. The solid lines are the responses at the natural frequency of the respective modes, and the dashed lines the unfiltered responses. 
converted into an equivalent erroneous rate measurement and compared to noise levels for a typical rate sensor.

When angular rate is measured, the $2 \theta$-drive mode is maintained at resonance and Coriolis coupling ensures that the response of the $2 \theta$-companion mode is proportional to the angular rate. For a sensor operating in the linear regime, the applied angular rate about the polar axis of the ring can be expressed as:

$$
\Omega_{z}=\frac{\hat{Q}_{s} \omega_{Q}^{*}}{2 \hat{Q}_{d} Q_{f, Q}}
$$

where $\omega_{Q}^{*}$ and $Q_{f, Q}$ are the natural frequency and $\mathrm{Q}$ factor for the $2 \theta$-modes including electrostatic effects, $\hat{Q}_{d}$ is the resonant amplitude of the $2 \theta$-drive mode, and $\hat{Q}_{s}$ is the resonant amplitude of the $2 \theta$-sense mode. Similar angular rate expressions can be obtained for the $3 \theta$-pair of coordinates.

Assuming the drive amplitude $\hat{Q}_{d}=2 \mu \mathrm{m}$ and the $\hat{Q}_{s}$ amplitude is the shock-induced response reported in Section 3.5, then the angular rate error measured with inner electrodes activated $\left(V_{i}=V_{o}\right)$ is:

$$
\Omega_{z}=-\frac{30 \times 10^{-9} \mathrm{~m} \times 2 \pi \times 17000 \mathrm{~Hz}}{2 \times 2 \times 10^{-6} \mathrm{~m} \times 60000}=-0.0134 \frac{\mathrm{rad}}{\mathrm{s}}=-0.765 \frac{\mathrm{deg}}{\mathrm{s}}
$$

Similar calculations can be performed for a sensor operating without the inner and outer electrodes activated $\left(V_{i}=0\right)$ and/or using the $3 \theta$-response to make rate measurements. Table 5 summarises the results obtained for the shock-induced rate errors for these cases.

\begin{tabular}{c|cc|cc} 
Mode & \multicolumn{2}{|c}{$V_{i}=0 \mathrm{~V}$} & \multicolumn{2}{c}{$V_{i}=V_{o}$} \\
& $\begin{array}{c}\text { Response } \\
\text { at } f_{(Q, R)}\end{array}$ & $\begin{array}{c}\text { Equivalent } \\
\text { rate }\end{array}$ & $\begin{array}{c}\text { Response } \\
\text { Equivalent } f_{(Q, R)}\end{array}$ & rate \\
\hline$Q$ & $535 \mathrm{~nm}$ & $13.64 \frac{\mathrm{deg}}{\mathrm{s}}$ & $30 \mathrm{~nm}$ & $0.77 \frac{\mathrm{deg}}{\mathrm{s}}$ \\
$R$ & $168 \mathrm{~nm}$ & $5.52 \frac{\mathrm{deg}}{\mathrm{s}}$ & $563 \mathrm{~nm}$ & $18.49 \frac{\mathrm{deg}}{\mathrm{s}}$ \\
\hline
\end{tabular}

Table 5: Modal responses and angular rate errors for considered scenarios

The calculated rate output can be compared to the noise level for a typical micro-ring rate sensor [2]. For an inductive MEMS ring-rate sensor, the noise level is reported to be $<0.04 \mathrm{deg} / \mathrm{s}$. Assuming similar noise levels apply to the capacitive devices analysed here, it clear that the $2 \theta$-shock induced responses exceed the reported noise-level indicating the possibility of erroneous rate measurement. The potential for this to occur is greatest when only the outer ring of electrodes is present $(13.64 \mathrm{deg} / \mathrm{s}>0.04 \mathrm{deg} / \mathrm{s})$. However, when inner electrodes are included the $2 \theta$-response is still significantly larger than the quoted noise level $(0.765 \mathrm{deg} / \mathrm{s}>0.04 \mathrm{deg} / \mathrm{s})$. It is also found that the $3 \theta$-response yields rate errors greater than the reported noise level for both electrode configurations, and the rate level with inner and outer electrodes activated is three-times the value obtained when outer electrodes only are activated. The reason for this large coupling is the proximity of the $3 \theta$-frequency to the $1 \theta+2 \theta$ frequency and the $3 \times 1 \theta$ frequency.

In summary, for the resonator designs considered, using only an outer ring of electrodes leads to sensors which are susceptible to shock when operating using $2 \theta$-modes 
of vibration, but this can be reduced significantly by including an inner ring of electrodes. Utilising $3 \theta$-modes of vibration, the shock susceptibility increases by a factor of three when inner and outer electrodes are used, compared to using outer electrodes only. For the sensor design considered, no electrode configuration yields responses close to or below reported levels of noise. The following section investigates how frequency separation affects the shock-induced angular rate error by considering the influence of changing frequency ratios $2 \theta / 1 \theta$ and $3 \theta / 1 \theta$.

\subsection{Frequency variation}

The results presented in earlier sections apply to one particular sensor design, with specific $1 \theta-, 2 \theta$ - and $3 \theta$-frequencies $\left(f_{P}, f_{Q}\right.$, and $f_{R}$ respectively). It was shown that the dominant coupling mechanism for exciting the $2 \theta$ - and $3 \theta$-coordinates under shock conditions is direct coupling from the $1 \theta$-coordinates. To understand the influence of frequency ratios $2 \theta / 1 \theta$ and $3 \theta / 1 \theta$ on the coupling, a parameter study is performed with the aim of identifying those frequency ratios that yield minimal coupling.

The parameter study involves varying the $f_{Q}$ and $f_{R}$ natural frequencies and calculating the resulting modal responses. The range of $2 \theta / 1 \theta$ - and $3 \theta / 1 \theta$-frequencies yielding parametric response (i.e. around $f_{Q}=2 f_{P}$ ) is excluded because parametric resonance is usually avoided in the design of rate sensors - although some studies have used parametric resonance to excite the resonator $[20,12]$. As such the behaviour at parametric response is not the focus of this paper. As in previous sections, numerical results are obtained with the inner electrodes activated and deactivated. The frequency ratios considered span the ranges $f_{Q} / f_{P}=1.2$ to 4.2 and $f_{R} / f_{P}=1.6$ to 5.2. These ranges cover a wide range of ring resonator designs and include the sensor design considered earlier, which corresponds to a frequency ratio $f_{Q} / f_{P}=1$.7. It is worthwhile noting that electrostatic softening behaviour is excluded for the frequency ratios considered, so the natural frequencies are calculated using the mechanical stiffnesses only.

Figure $15 \mathrm{a}$ shows the response at the $2 \theta$-frequency to shock for different ratios of $2 \theta / 1 \theta$-frequency, and Figure $15 \mathrm{~b}$ shows the response at the $3 \theta$-frequency to shock for different ratios of $3 \theta / 1 \theta$-frequency. In both figures, the solid line shows the response with inner electrodes deactivated, and the dashed line shows the response with inner electrodes having the same voltage as the outer electrodes. In these results, $9^{\text {th }}$ order electrostatic generalised forcing functions are used.

The results indicate that having a $2 \theta$ natural frequency that is much greater than twice the $1 \theta$ natural frequency yields reduced levels of shock induced coupling to the $2 \theta$-response. This trend is clear when the outer electrodes only are activated $\left(V_{i}=0\right)$ and when both the outer and inner electrodes are active $\left(V_{i}=V_{o}\right)$. Activating the inner electrodes has the effect of reducing the coupling and the observed $2 \theta$-response levels over the range of parameters considered. In this case, the reduction achieved is approximately $97.5 \%$. The design considered earlier has a frequency ratio $f_{Q} / f_{P}=1.7$ and is less favourable as the response levels are generally higher for frequency ratios $<2$.

For the 30-response, large response values are expected to occur for frequency ratios corresponding to parametric resonance, as cubic electrostatic direct-forcing terms induce response peaks when $f_{R} / f_{P}=3$. The observed response peaks are at slightly lower frequency ratios because linear and nonlinear electrostatic softening reduces the resonant frequencies. In contrast to the $2 \theta$-response, inner electrode activation increases the re- 


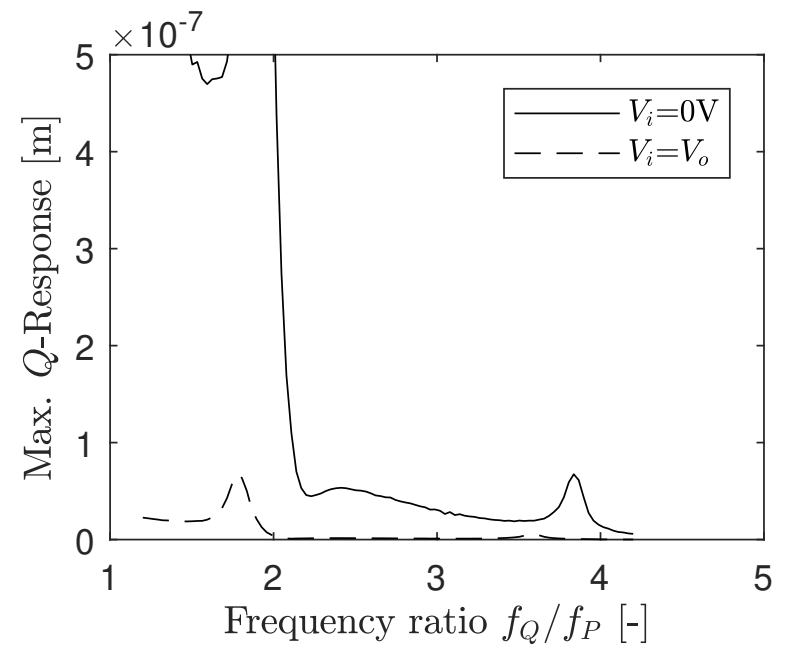

(a)

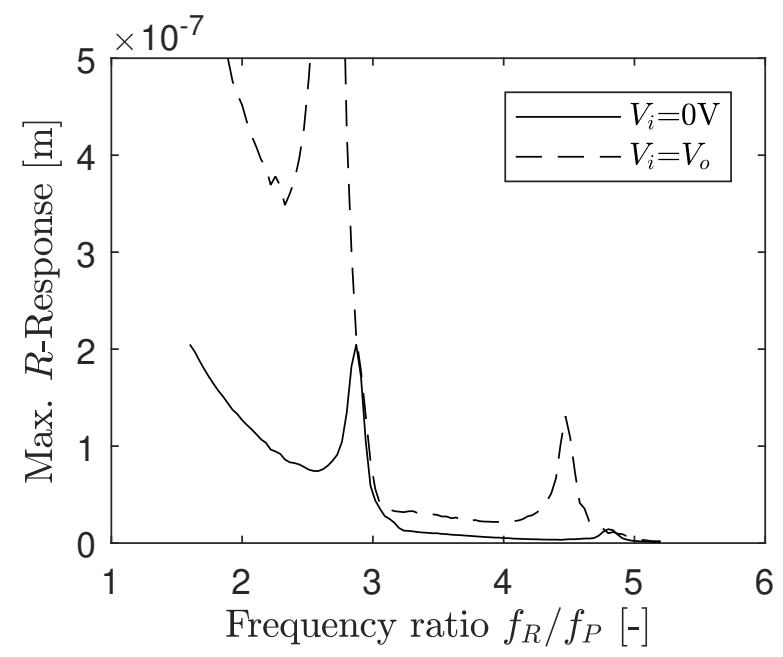

(b)

Figure 15: Shock response at the $2 \theta$ - and $3 \theta$-frequency for different ratios of $f_{Q} / f_{P}$ and $f_{R} / f_{P}$, respectively. The solid line describes the response with outer electrodes only, and the dashed line the response when the inner and outer electrodes have equal potential.

sponse for $3 \theta$-responses by a factor of $>3$. Further parametric resonances are expected to occur and are caused by higher order electrostatic nonlinear force terms.

Figure 16 shows the equivalent rate output of the filtered response in Figure 15 . The $2 \theta-$ and $3 \theta$-response are filtered with elliptical band-pass filters with the natural frequencies at the centre of the pass-band frequency range. The equivalent rate is calculated from the filtered flexural responses using Equation (26). The obtained rate output in the case of $V_{i}=0$ for $2 \theta$-responses (Figure 16a) becomes comparatively large for frequency ratios $f_{Q} / f_{P}$ approaching 2 , and ratios of $f_{R} / f_{P}$ approaching 3 as the conditions necessary for parametric response occur. The general level of erroneous rate output decreases for smaller or larger frequency ratios. In the range $f_{Q} / f_{P}<2$, minimum coupling is achieved for $f_{Q} / f_{P}=1.32$ with a rate output of approx. $0.43 \mathrm{deg} / \mathrm{s}$. For frequency ratios above two, the rate output error decreases sharply to values less than the reported noise level before reaching a peak caused by higher order parametric responses close to $f_{Q} / f_{P}=4$. When the inner electrodes are deactivated, the rate output exceeds the reported noise level for all considered frequency ratios and falls below $0.5 \mathrm{deg} / \mathrm{s}$ only when $2 \theta / 1 \theta$-ratios $>4$.

For the $3 \theta$ rate response with the inner electrodes are deactivated, the minimum rate response below $f_{R} / f_{P}=3$ is $2.76 \mathrm{deg} / \mathrm{s}$ which occurs when $f_{R} / f_{P}=2.53$. Above the first parametric resonance peak, the response level falls significantly to about $0.18 \mathrm{deg} / \mathrm{s}$ when $f_{R} / f_{P}=4.50$ with a small peak occurring close to $f_{R} / f_{P}=5$. When the inner electrodes are active the responses are significantly larger and the minimum rate output is $1.35 \mathrm{deg} / \mathrm{s}$ when $f_{R} / f_{P}=3.90$. Below a frequency ratio of $3 \theta / 1 \theta=3$, the minimum rate output is $13.6 \mathrm{deg} / \mathrm{s}$ when $f_{R} / f_{P}=2.25$.

In summary, the shock induced rate output error is negligible for the $2 \theta$-response over quite a large range of frequency ratios above 2 provided the inner electrodes are active and there is no parametric resonance. This indicates that the effects of shock can 


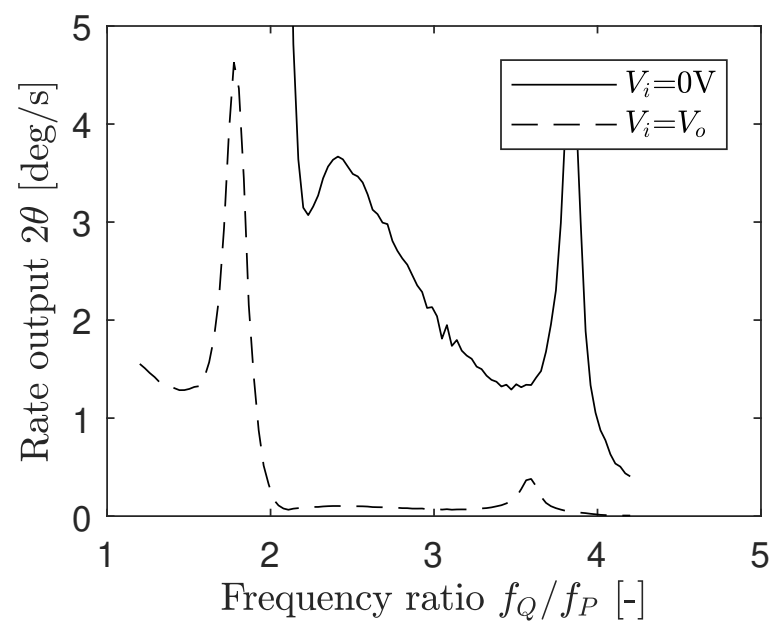

(a)

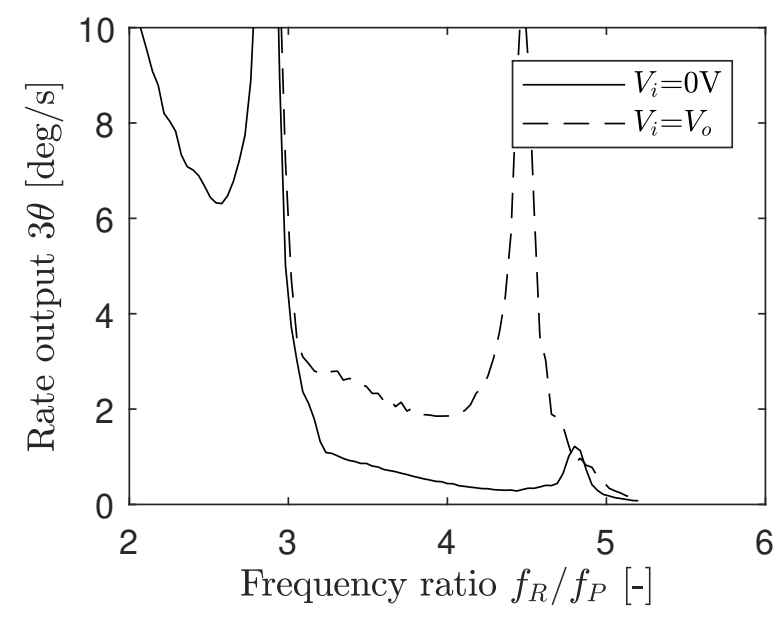

(b)

Figure 16: Calculated rate outputs from responses in Figure 15. The graph with the solid line describes the response with outer electrodes only, and the graph with the dashed line the response when the inner and outer electrodes have equal potential.

be mitigated by properly spacing the $2 \theta$ - and $1 \theta$-frequencies to design a resonator that minimises coupling between $1 \theta$ - and $2 \theta$-responses. In contrast, the results obtained for the $3 \theta$-response indicate that the coupling is significant over the frequency range considered with or without the inner electrodes activated and that devices operating using $3 \theta$-modes are more susceptible to shock than devices operating using $2 \theta$-modes.

\section{Conclusion}

An analytical model was developed to investigate the effects of shock on the dynamic behaviour of a capacitive ring based CVG with nonlinear electrostatic forcing. The model describes the ring response in terms of the modes of a perfect ring and illustrates how nonlinear electrostatic forces induce mode coupling. The nonlinear coupling of rigidbody to flexural motions was demonstrated and direct-forcing terms were identified as the dominant coupling terms. Shock induced mode coupling can have a detrimental affect on device performance by producing erroneous angular rate measurements. For devices operating using $2 \theta$-modes, it was found that severe shock can induce high levels of coupling if electrodes are only present outside the ring, but the coupling is reduced significantly when electrodes are included inside the ring. In contrast, for devices operating using $3 \theta$-modes, the coupling increases when inner electrodes are used. Parameter studies were performed to minimise the mode coupling by modifying the frequency separation as a ratio and it was found that the coupling could be reduced further in $2 \theta$-devices by carefully selecting the spacing between $2 \theta$ - and $1 \theta$-frequencies, and avoiding parametric resonance. For the range of frequencies considered it was found that devices operating using $3 \theta$-modes are more susceptible to shock than devices operating using $2 \theta$-modes. 


\section{Appendix A. Energy calculations}

For the electrostatic energy $E_{\text {cap }}$, the intermediate calculations performed to yield the final expressions are provided in this appendix. The expressions for the electrostatic energies of the outer and inner rings of capacitors are given in Section 2.3. Drawing the integrals in the parenthesis gives

$$
\begin{aligned}
& E_{\text {cap }, o}=\frac{\varepsilon_{0} \varepsilon V_{o}^{2} l r_{o}}{2 d_{g}}\left[\int_{0}^{2 \pi} 1 \mathrm{~d} \varphi+\int_{0}^{2 \pi} \frac{w}{d_{g}} \mathrm{~d} \varphi+\int_{0}^{2 \pi}\left(\frac{w}{d_{g}}\right)^{2} \mathrm{~d} \varphi+\int_{0}^{2 \pi}\left(\frac{w}{d_{g}}\right)^{3} \mathrm{~d} \varphi+\cdots\right] \\
& E_{\text {cap }, i}=\frac{\varepsilon_{0} \varepsilon V_{i}^{2} l r_{i}}{2 d_{g}}\left[\int_{0}^{2 \pi} 1 \mathrm{~d} \varphi-\int_{0}^{2 \pi} \frac{w}{d_{g}} \mathrm{~d} \varphi+\int_{0}^{2 \pi}\left(\frac{w}{d_{g}}\right)^{2} \mathrm{~d} \varphi-\int_{0}^{2 \pi}\left(\frac{w}{d_{g}}\right)^{3} \mathrm{~d} \varphi+-\cdots\right]
\end{aligned}
$$

where the integration of 1 with respect to the ring angle gives $2 \pi$. Each fraction $\left(w / d_{g}\right)^{(p+1)}$ can be evaluated separately, and is identified by the degree of electrostatic accuracy $p$. To evaluate, which terms yield non-zero contributions to the final energy expression when integrated with respect to the angle $\varphi$ over 0 to $2 \pi$, the radial displacement $w$ is substituted with the generalised coordinates.

The single integrals for the calculation of the electrostatic energy expression include multiples and powers of the mode shape functions of the generalised coordinates, and yield

$$
\begin{aligned}
\int_{0}^{2 \pi}\left(\frac{w}{d_{g}}\right)^{(p+1)} \mathrm{d} \varphi= & \frac{1}{d_{g}{ }^{(p+1)}} \int_{0}^{2 \pi}\left[P_{1} \cos (\varphi)+P_{2} \sin (\varphi)+Q_{1} \cos (2 \varphi) \cdots\right. \\
& \left.+Q_{2} \sin (2 \varphi)+R_{1} \cos (3 \varphi)+R_{2} \sin (3 \varphi)\right]^{(p+1)} \mathrm{d} \varphi
\end{aligned}
$$

For $p=0$, the integrals of all trigonometric functions yield zero. This means, the static forcing on the generalised coordinates is zero for all considered coordinates.

The $p=1$ terms yield multiples of the trigonometric functions, which yields

$$
S_{2}=\int_{0}^{2 \pi}\left(\frac{w}{d_{g}}\right)^{2} \mathrm{~d} \varphi=\frac{\pi}{d_{g}{ }^{2}}\left[{\dot{P_{1}}}^{2}+{\dot{P_{2}}}^{2}+{\dot{Q_{1}}}^{2}+{\dot{Q_{2}}}^{2}+{\dot{R_{1}}}^{2}+{\dot{R_{2}}}^{2}\right]
$$

These terms yield linear electrostatic forces when the derivative with respect to the generalised coordinates is calculated.

The nonlinear $p=2$ and $p=3$ terms contain cubic and quartic terms, with products of three trigonometric functions. There are an increasing number of terms, yielding a large number of possible trigonometric combinations. However, a large part of these combinations leads to zero on evaluation of the integral. The integrals are evaluated 
symbolically and result in the electrostatic energies

$$
\begin{aligned}
S_{3}=\int_{0}^{2 \pi}\left(\frac{w}{d_{g}}\right)^{3} \mathrm{~d} \varphi= & \frac{3 \pi}{2 d_{g}{ }^{3}}\left[-Q_{1} P_{2}^{2}+Q_{1} P_{1}^{2}+2 Q_{1} R_{1} P_{1} \cdots\right. \\
& \left.+2 Q_{2} P_{1} P_{2}-2 R_{1} Q_{2} P_{2}+2 Q_{1} R_{2} P_{2}+2 Q_{2} R_{2} P_{1}\right]
\end{aligned}
$$

and

$$
\begin{aligned}
& S_{4}=\int_{0}^{2 \pi}\left(\frac{w}{d_{g}}\right)^{4} \mathrm{~d} \varphi=\frac{\pi}{4 d_{g}{ }^{4}}\left[12 Q_{1}{ }^{2} R_{1}{ }^{2}+3 R_{2}{ }^{4}+12 Q_{2}{ }^{2} R_{2}{ }^{2}+12 Q_{1}{ }^{2} R_{2}{ }^{2}+6 R_{1}{ }^{2} R_{2}{ }^{2} \ldots\right. \\
& +12 Q_{2}{ }^{2} R_{2} P_{2}+3 Q_{1}{ }^{4}+3 R_{1}{ }^{4}+3 Q_{2}{ }^{4}+3 P_{2}{ }^{4}+3 P_{1}{ }^{4}+12 P_{1}{ }^{2} R_{2} P_{2} \ldots \\
& +24 Q_{2} R_{2} Q_{1} P_{1}+24 Q_{1} R_{1} Q_{2} P_{2}-12 R_{1} P_{1} Q_{2}{ }^{2}-12 Q_{1}{ }^{2} R_{2} P_{2} \ldots \\
& -12 R_{1} P_{1} P_{2}^{2}+12 Q_{1}^{2} R_{1} P_{1}+12 R_{1}^{2}{Q_{2}}^{2}+12 R_{2}{ }^{2} P_{1}{ }^{2}+6 Q_{1}{ }^{2} Q_{2}{ }^{2} \cdots \\
& +12 R_{1}{ }^{2} P_{2}{ }^{2}+12 P_{1}{ }^{2} Q_{2}{ }^{2}+12 R_{2}{ }^{2} P_{2}{ }^{2}-4 P_{2}^{3} R_{2}+12 P_{2}{ }^{2} Q_{2}{ }^{2} \cdots \\
& \left.+12 Q_{1}{ }^{2} P_{2}{ }^{2}+6 P_{1}^{2} P_{2}^{2}+12 R_{1}^{2} P_{1}^{2}+4 P_{1}^{3} R_{1}+12 P_{1}^{2} Q_{1}{ }^{2}\right]
\end{aligned}
$$

Substituting Equations (A.2) to (A.4) into Equations (A.1a) and (A.1b) yield the outer and inner electrostatic energies.

\section{References}

[1] M. Amabili and M.P. Paidoussis. Review of studies on geometrically nonlinear vibrations and dynamics of circular cylindrical shells and panels, with and without fluid-structure interaction. Appl Mech Reviews, 56(4):349-382, 2003.

[2] F. Ayazi and K. Najafi. A HARPSS polysilicon vibrating ring gyroscope. J Microelectromech Syst, 10:169-179, 2001.

[3] S. Chang, M. Chia, P. Castillo-Borelley, W. Higdon, Q. Jiang, J. Johnson, L. Obedier, M. Putty, Q. Shi, D. Sparks, and S. Zarabadi. An electroformed cmos integrated angular rate sensor. Sens Actuators A Phys, 66:138-143, 1998.

[4] B. Chouvion, C.H.J. Fox, S. McWilliam, and A.A. Popov. In-plane free vibration analysis of combined ring-beam structural systems by wave propagation. J Sound Vib, 329:5087-5104, 2010.

[5] B. Chouvion, S. McWilliam, and A.A. Popov. Effect of nonlinear electrostatic forces on the dynamic behaviour of a capacitive ring-based coriolis vibrating gyroscope under severe shock. MSSP, in press, 2017.

[6] A. Duwel, J. Gorman, M. Weinstein, J. Borenstein, and P. Ward. Experimental study of thermoelastic damping in MEMS gyros. Sens Actuators A Phys, 103:70-75, 2003. 
[7] R. Eley, C.H.J. Fox, and S. McWilliam. The dynamics of a vibrating-ring multi-axis rate gyroscope. Proc. Inst. Mech. Eng. C J. Mech. Eng. Sci., 214:1503-1513, 2000.

[8] C.H.J. Fox. A simple theory for the analysis and correction of frequency splitting in slightly imperfect rings. J Sound Vib, 142(2):227-242, 1990.

[9] C.H.J. Fox and D.J.W. Hardie. Vibratory gyroscopic sensors. In DGON Symposium on Gyro Technology, Stuttgart, September 11,12, pages 130-133, 1984.

[10] C.H.J. Fox, R.S. Hwang, and S. McWilliam. The in-plane vibration of thin rings with in-plane profile variations part 2: Application to nominally circular rings. $J$ Sound Vib, 220(3):517-539, 1999.

[11] B.J. Gallacher, J. Hedley, J.S. Burdess, A.J. Harris, A. Rickard, and D.O. King. Electrostatic correction of structural imperfections present in a microring gyroscope. J Microelectromech Syst, 14(2):221-234, 2005.

[12] K.M. Harish, B.J. Gallacher, J.S. Burdess, and J.A. Neasham. Simple parametric resonance in an electrostatically actuated microelectromechanical gyroscope. In Proc Inst Mech Eng C J Mech Eng Sci, pages 43-52, 2008.

[13] C.M. Harris and A.G. Piersol, Editors. Harris' Shock and Vibration Handbook. McGraw-Hill, New York, 5th edition, 2002.

[14] S.T. Hossain, S. McWilliam, and A.A. Poov. An investigation on thermoelastic damping of high-q ring resonators. Int. J. of Mech. Sci., 106:209-219, 2016.

[15] R.S. Hwang, C.H.J. Fox, and S. McWilliam. The in-plane vibration of thin rings with in-plane profile variations part 1: General background and theoretical formulation. J Sound Vib, 220(3):497-516, 1999.

[16] M.I. Ibrahim, M.I. Younis, and F. Alsaleem. An investigation into the effects of electrostatic and squeeze-film non-linearities on the shock spectrum of microstructures. Int J Non Linear Mech, 45:756-765, 2010.

[17] S.T. Karris. Signals and Systems: With MATLAB Computing and Simulink Modeling. Orchard Publications, Fremont, CA, USA, 2012.

[18] S. McWilliam, J. Ong, and C. Fox. On the statistics of natural frequency splitting for rings with random mass imperfections. J. Sound Vib., 279:453-470, 2003.

[19] A.H. Ramini, M.I. Younis, and R. Miles. Modeling the effects of pcb motion on the response of microstructures under mechanical shock. J. Vib. Acoust., 133:9 pages, 2011.

[20] J.F. Rhoads, S.W. Shaw, and K.L. Turner. Nonlinear dynamics and its applications in micro- and nanoresonators. J Dyn Syst Meas Control, 132(3):1-14, 2010.

[21] A.K. Rourke, S. McWilliam, and C.H.J. Fox. Frequency trimming of a vibrating ring-based multi-axis rate sensor. J Sound Vib, 280:495-530, 2005. 
[22] S.D. Senturia. Microsystem Design. Kluwer Academic Publishers, Norwell, USA, 2001.

[23] G.B.D. Serandour, C.H.J. Fox, and S. McWilliam. Non-linear in-plane vibrations of coriolis based gyroscope. In Proc 2004 Int Conf Noise and Vib Eng, ISMA, Sept 20-22, 2004, Leuven, Belgium, pages 2183-2193, 2004.

[24] S. Sieberer. In-plane Shock Response of Capacitive MEMS Ring-rate Sensors. PhD thesis, The University of Nottingham, 2014.

[25] S. Sieberer, A. A. Popov, and S. McWilliam. Shock-induced electrostatic coupling of modes of vibration in the response of a MEMS ring sensor. In Proc ASME 2012 IDETC \& CIE Conference, August 12-15, 2012, Chicago, IL, USA.

[26] C. Song and M. Shinn. Commercial vision of silicon-based inertial sensors. Sens Actuators A Phys, 66:231-236, 1998.

[27] V.T. Srikar and S.D. Senturia. The reliability of microelectromechanical systems (MEMS) in shock environments. J Microelectromech Syst, 11(3):206-214, 2002.

[28] J.W. Strutt Lord Rayleigh. The Theory of Sound, volume 1. MacMillan and Co, London, 1877.

[29] S. Sundaram, M. Tormen, B. Timotijevic, R. Lockhart, T. Overstoly, R.P. Stanley, and H.R. Shea. Vibration and shock reliability of mems: modeling and experimental validation. J Micromech Microeng, 21(4), 2011.

[30] S.A. Tobias. A theory of imperfection for the vibrations of elastic bodies of revolution. Engineering, 172(4470):409-410, 1951.

[31] W. Weaver, S.P. Timoshenko, and D.H. Young. Vibration Problems in Engineering. John Wiley and Sons, 5th edition, 1990.

[32] S.J. Wong, C.H.J. Fox, and S. McWilliam. Thermoelastic damping of the in-plane vibration of thin silicon rings. J Sound Vib, 293:266-285, 2006.

[33] S. Yoon, U. Park, R. Rhim, and S. Yang. Tactical grade mems vibrating ring gyroscope with high shock sensitivity. Microelectronics Engineering, 142:22-29, 2015.

[34] S.W. Yoon, S. Lee, and K. Najafi. Vibration sensitivity analysis of MEMS vibratory ring gyroscopes. Sens Actuators A Phys, 171:163-177, 2011.

[35] M.I. Younis, F. Alsaleem, and D. Jordy. The response of clamped-clamped microbeams under mechanical shock. Int J Non Linear Mech, 42:643-657, 2007.

[36] M.I. Younis, R. Miles, and D. Jordy. Investigation of the response of microstructures under the combined effect of mechanical shock and electrostatic forces. J Micromech Microeng, 16:2463-2474, 2006.

[37] M.I. Younis and A.H. Nayfeh. A study of the nonlinear response of a resonant microbeam to an electric actuation. Nonlinear Dyn, 31:91-117, 2003. 